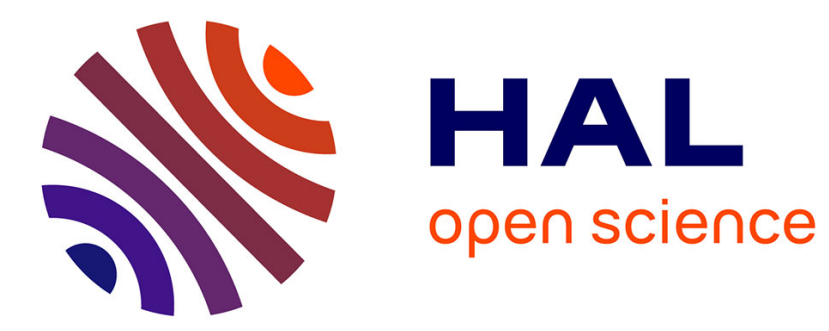

\title{
Recursive Finite-Difference Lattice Boltzmann Schemes
}

\author{
Lucien Vienne, Emmanuel Lévêque
}

\section{To cite this version:}

Lucien Vienne, Emmanuel Lévêque. Recursive Finite-Difference Lattice Boltzmann Schemes. Computers \& Mathematics with Applications, 2021, 96, pp.95-108. 10.1016/j.camwa.2021.05.016 . hal$03118913 \mathrm{v} 2$

\section{HAL Id: hal-03118913 \\ https://hal.science/hal-03118913v2}

Submitted on 26 May 2021

HAL is a multi-disciplinary open access archive for the deposit and dissemination of scientific research documents, whether they are published or not. The documents may come from teaching and research institutions in France or abroad, or from public or private research centers.
L'archive ouverte pluridisciplinaire HAL, est destinée au dépôt et à la diffusion de documents scientifiques de niveau recherche, publiés ou non, émanant des établissements d'enseignement et de recherche français ou étrangers, des laboratoires publics ou privés.

\section{(이)(\$)}

Distributed under a Creative Commons Attribution - NonCommercial - NoDerivatives| 4.0 


\title{
Recursive Finite-Difference Lattice Boltzmann Schemes*
}

\author{
Lucien Vienne*, Emmanuel Lévêque \\ Laboratoire de Mécanique des Fluides et d'Acoustique \\ Univ Lyon, Ecole Centrale de Lyon, CNRS, Univ Claude Bernard Lyon 1, INSA Lyon, LMFA, UMR5509, 69130, Ecully, \\ France
}

\begin{abstract}
The motivation of this study is twofold. First, a recursive mathematical formulation of the discrete-velocity Boltzmann equation (DVBE) under the Bhatnagar-Gross-Krook (BGK) approximation is introduced. This formulation allows us to formally express the solution of the DVBE as an infinite sum over successive particle derivatives of the distributions associated with local equilibrium. A Chapman-Enskog multiplescales expansion shows that this sum can be safely truncated beyond the second order if the Navier-Stokes level of description is requested. Therefore, the distribution functions depend only on the first and secondorder derivatives of the related equilibrium distributions. This alternative equation to the DVBE defines a basis to design kinetic schemes for the evolution of the distribution functions based solely on flow variables that are sufficient to define local equilibrium. Second, a family of mass-conserving numerical schemes is introduced from this kinetic equation by discretizing the particle derivatives by backward finite differences. Interestingly, a so-called "simplified Lattice Boltzmann method" introduced by Chen et al. in 2017 can be recast in this family. Numerical simulations highlight a level of numerical dissipation that is generally higher than the level obtained with a standard Lattice Boltzmann scheme, as expected by approximating derivatives by finite differences. Nevertheless, we show by using a von Neumann analysis that it is possible to parametrize our scheme, according to the relaxation coefficient of the DVBE, to reduce significantly its numerical dissipation and improve its spectral properties.

We believe that this modeling can also be of interest to connect macroscopic and kinetic representations, e.g. when dealing with initial and boundary conditions or in hybrid simulations matching Navier-Stokes and Lattice Boltzmann schemes.
\end{abstract}

Keywords: Lattice Boltzmann method, recursive formulation, finite difference, von Neumann analysis 2020 MSC: 76P05, 76M20, 35Q20

\section{Introduction}

The Lattice Boltzmann (LB) method follows from a discretization of the Boltzmann equation to solve weakly-compressible fluid dynamics [1, 2. Using a kinetic approach to simulate continuum flows seems at first glance unreasonable but, nevertheless, has certain advantages. At first, the velocity space is amenable to a radical decimation so that only a small set of microscopic velocities, e.g. nine in two dimensions, is sufficient to reconstruct the Navier-Stokes dynamics at the macroscopic level. In addition, non-locality and non-linearity are disentangled in the kinetic equations, which facilitates numerical integration 3 . As a result, the so-called stream-and-collide LB algorithm is simple, accurate and formidably efficient in terms of computations 4 .

${ }^{\star}$ https://doi.org/10.1016/j.camwa.2021.05.016 (C) 2021. This manuscript version is made available under the CC-BYNC-ND 4.0 license http://creativecommons.org/licenses/by-nc-nd/4.0/

${ }^{*}$ Corresponding author

Email address: contact@lvienne.com (Lucien Vienne) 
In the LB approach, the degrees of freedom, or nodal values, refer to the distribution functions $\left(f_{0}, \cdots, f_{N-1}\right)$ of particles with the microscopic velocities $\left(\mathbf{c}_{0}, \cdots, \mathbf{c}_{N-1}\right)$. The scheme governs the evolution in time of these distributions at each lattice node. At the macroscopic level, flow variables are recovered by summing the contributions from the different (microscopic) velocities so that

$$
\rho=\sum_{\alpha=0}^{N-1} f_{\alpha} \quad \text { and } \quad \rho \boldsymbol{u}=\sum_{\alpha=0}^{N-1} f_{\alpha} \boldsymbol{c}_{\alpha},
$$

where $\rho$ and $\mathbf{u}$ denote respectively the mass density and the velocity of the fluid. Even if the decimation in velocity space is drastic, the number of nodal values remains significantly higher that the number of reconstructed flow variables, e.g. in two dimensions nine distribution functions are required to reconstruct locally the mass density and the two components of the velocity. The difference is even more pronounced in three-dimensions where nineteen or twenty seven densities are required. This overload of information is problematic when a given macroscopic solution needs to be prescribed at the mesoscopic level, e.g. for initial or boundary conditions. In that case, the kinetic solution is indeterminate and ad-hoc constraints must be invoked [2. From a computational viewpoint, the LB algorithm is data intensive and memory bound, which can be detrimental to its portability on accelerators such as Graphical Processing Units (GPUs). In this article, a LB algorithm that relies on flow variables only, and therefore requires less degrees of freedom, is introduced. It stems from the truncation and the discretization of a recursive formulation of the discretevelocity Boltzmann equation (DVBE).

Some variants of the original stream-and-collide algorithm, depending on flow variables only, have already been proposed. In particular, Inamuro suggested a Bhatnagar-Gross-Krook (BGK) collision with a relaxation time (towards statistical equilibrium) equal to the time step of the algorithm. Therefore, distributions are considered at equilibrium and depend only on flow variables $(\rho, \mathbf{u})[5$. This strong assumption gives a nonphysical value to the fluid viscosity that can then be corrected by adding a contribution of the viscous stress tensor estimated by finite differences. Asinari et al. derived a link-wise artificial compressibility scheme that can be formulated in the LB framework, with a collision operator that is non-local but depends on distributions at equilibrium only [6]. Let us mention that the scheme developed by Inamuro can be viewed as a special case of the link-wise artificial compressibility scheme. More recently, Chen et al. suggested to consider the equilibrium state at the previous time step to build a prediction of the solution, which is afterwards corrected [7] by considering a first-order approximation (in Knudsen number) of the DVBE. This prediction-correction scheme only involves macroscopic quantities. We shall see that this last scheme fits into the general framework developed in the present study.

A key ingredient behind formulating a LB scheme that relies only on flow variables is to express the nonequilibrium component of the distributions, $f_{i}^{n e q} \equiv f_{i}-f_{i}^{e q}$, as a function of the space-and-time derivatives of the equilibrium component $f_{i}^{e q}(\rho, \mathbf{u})$. In this regard, Holdych et al. 8] showed that $f_{i}^{n e q}$ could be rewritten in a recursive manner as

$$
f_{\alpha}^{n e q}=\sum_{m=1}^{\infty}(-\tau)^{m}\left(\partial_{t}+\boldsymbol{c}_{\alpha} \cdot \nabla\right)^{m} f_{\alpha}^{e q} .
$$

Their motivation was to derive the truncation errors of this expansion and to show that some errors cancel out for specific values of the relaxation time $\tau$. This iterative approach is similar to the so-called "Maxwell iteration" for the LB method [9], in which the macroscopic equations are derived without explicitly resorting to a multiple-scale Chapman-Enskog expansion 2. Finally, it has also been used to tailor the equilibrium function of the standard LB scheme to solve non-linear equations such as the Burgers, Korteweg-de-Vries or Kuramoto-Sivashinsky equations by Otomo et al. [10. In the present article, our contribution is to use Eq. (2) to derive original LB schemes which rely on flow variables only. This is made possible by first showing that the sum can actually be truncated at $m=2$ to comply with the Navier-Stokes dynamics at the macroscopic level. Then, numerical schemes can be designed by finite-difference discretization of the first and second-order particle derivatives.

The remainder of the paper is organized as follows. Section 2 introduces the recursive formulation of the discrete-velocity Boltzmann equation and its discretization by finite differences. An original finite-difference 
LB scheme is proposed. It is also shown that the aforementioned prediction-correction scheme by Chen et al. [7] can be derived in this framework. In section 3] a detailed comparison with the classical LB scheme is performed on the double shear layer and Taylor-Green vortex two-dimensional flows. Section 4 gives further insight on the proposed scheme by exploring spectral properties with a von-Neumann analysis. Finally, concluding remarks and perspectives are drawn in Section 5 .

\section{On a recursive formulation of the discrete-velocity Boltzmann equation}

\subsection{Context and derivation of the recursive formulation}

In the LB approach, the sums replace the integrals in the statistical moments, as expressed in Eq. (1). This discretization of the velocity space stems from expanding and truncating the solution of the continuum Boltzmann equation onto a finite basis of Hermite polynomials in velocity, and resorting to a Gaussian quadrature formula to express the statistical moments [1, 11. Therefore, the discrete set of velocities may be thought of as the nodes in the Gaussian quadrature formula. It can then be established that the distributions $f_{\alpha}(\mathbf{x}, t)$ evolve according to a discrete-velocity analogue of the Boltzmann equation

$$
\left(\partial_{t}+c_{\alpha} \cdot \nabla\right) f_{\alpha}=-\frac{1}{\tau}\left(f_{\alpha}-f_{\alpha}^{e q}\right),
$$

where the BGK approximation to the collision [12] is used in the right-hand side. This approximation expresses as a relaxation of the distributions towards their values at equilibrium, $f_{i}^{e q}(\rho, \mathbf{u})$, with a unique relaxation time $\tau=\nu / c_{s}^{2}$ fixed by the kinematic fluid viscosity $\nu$ and the speed of sound $c_{s}$. This is sufficient to ensure that the slowly varying solution of the hierarchy of statistical equations built from Eq. (3) satisfy the isothermal Navier-Stokes equations in the low-Mach-number limit.

The set of microscopic velocities $\left(\mathbf{c}_{0}, \cdots, \mathbf{c}_{N-1}\right)$ determine the lattice so that the particles are streamed from one node to a neighbouring node during exactly one time step. In the present study, numerical results will refer to the two-dimensional D2Q9 lattice associated with the set of velocities $\left(\mathbf{c}_{0}, \cdots, \mathbf{c}_{8}\right)$ given by the Cartesian components (see Fig. 1)

$$
\boldsymbol{c}_{\alpha}=\left[\begin{array}{ccccccccc}
0 & 1 & 0 & -1 & 0 & 1 & -1 & -1 & 1 \\
0 & 0 & 1 & 0 & -1 & 1 & 1 & -1 & -1
\end{array}\right]^{T}
$$

in lattice units 1

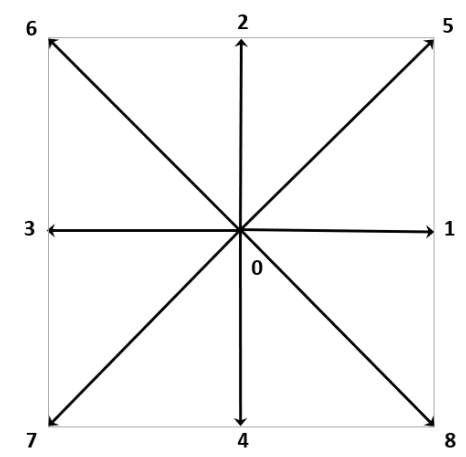

Figure 1: Set of velocities of the D2Q9 lattice.

The equilibrium distribution in Eq. (3) is an essential ingredient of the kinetic representation. In theory, it refers to a (continuous) Maxwell-Boltzmann distribution for classical fluids. In the LB framework, it is

\footnotetext{
${ }^{1}$ Lattice units are obtained by normalizing space and time by the lattice spacing and the time step of the algorithm, respectively.
} 
replaced by its Hermite expansion, which is commonly truncated at the second order. For our D2Q9 lattice, it yields

$$
f_{\alpha}^{e q}(\rho, \boldsymbol{u})=w_{\alpha} \rho\left[1+\frac{\boldsymbol{c}_{\alpha} \cdot \boldsymbol{u}}{c_{s}{ }^{2}}+\frac{\left(\boldsymbol{c}_{\alpha} \cdot \boldsymbol{u}\right)^{2}}{2 c_{s}{ }^{4}}-\frac{\boldsymbol{u}^{2}}{2 c_{s}{ }^{2}}\right]
$$

where the weights $w_{\alpha}$ are related to the lattice connectivity with $w_{0}=4 / 9, w_{1 \ldots 4}=1 / 9$ and $w_{5 \ldots 8}=1 / 36$ (see Fig. 1).

Let us now return to the discrete-velocity Boltzmann equation (3) which can be rewritten as

$$
f_{\alpha}=f_{\alpha}^{e q}-\tau\left(\partial_{t}+\boldsymbol{c}_{\alpha} \cdot \nabla\right) f_{\alpha} .
$$

The recursive formulation arises by replacing $f_{\alpha}$ in the rhs of Eq. (6) by its expression and by repeating this procedure infinitely. This leads formally to the expansion

$$
f_{\alpha}=f_{\alpha}^{e q}+\sum_{m=1}^{\infty}(-\tau)^{m}\left(\partial_{t}+\boldsymbol{c}_{\alpha} \cdot \boldsymbol{\nabla}\right)^{m} f_{\alpha}^{e q}
$$

in substitution of Eq. (3). This formulation shows that the $f_{\alpha}$ 's can be expressed in terms of flow variables $(\rho, \mathbf{u})$, however, this comes at the price of an infinite sum of increasing-order derivatives. The BGK collision operator is hidden in the form of this equation. Interestingly, the distribution function expresses (in lattice units) as an expansion in powers of the small parameter $\bar{\tau}=\tau / \delta_{t}$,

$$
f_{\alpha}=f_{\alpha}^{(e q)}(\rho, \mathbf{u})-\bar{\tau}\left(\partial_{t}+\boldsymbol{c}_{\alpha} \cdot \boldsymbol{\nabla}\right) f_{\alpha}^{(e q)}(\rho, \mathbf{u})+\bar{\tau}^{2}\left(\partial_{t}+\boldsymbol{c}_{\alpha} \cdot \boldsymbol{\nabla}\right)^{2} f_{\alpha}^{(e q)}(\rho, \mathbf{u})+\ldots
$$

At this stage, one might wonder whether this infinite sum could be truncated. We will demonstrate through a multiple-timescales Chapman-Enskog expansion that it is sufficient to consider the first two terms in the sum to comply with the Navier-Stokes equations at the macroscopic level.

\subsection{Multiple-timescales Chapman-Enskog expansion}

Macroscopic fluid dynamics follows from seeking solutions (to the hierarchy of statistical equations) that vary on a much slower timescale than the collisional timescale $\tau$. This is usually done by using a multiple-timescales Chapman-Enskog expansion in the small parameter $\epsilon$, with

$$
\begin{aligned}
f_{\alpha} & =f_{\alpha}^{(0)}+\epsilon f_{\alpha}^{(1)}++\epsilon^{2} f_{\alpha}^{(2)}+\cdots \\
\partial_{t} & =\epsilon \partial_{t_{1}}+\epsilon^{2} \partial_{t_{2}}+\cdots \\
\nabla & =\epsilon \nabla_{1}
\end{aligned}
$$

The parameter $\epsilon$ may be identified physically with the Knudsen number and, $t_{1}$ and $t_{2}$ with advective and diffusive timescales. Substituting these expansions into our recursive formulation, we obtain at successive orders in $\epsilon$

$$
\begin{gathered}
O\left(\epsilon^{0}\right): \quad f_{\alpha}^{(0)}=f_{\alpha}^{e q} \\
O\left(\epsilon^{1}\right): \quad f_{\alpha}^{(1)}=-\tau\left(\partial_{t_{1}}+\boldsymbol{c}_{\alpha} \cdot \nabla_{1}\right) f_{\alpha}^{(0)} \\
O\left(\epsilon^{2}\right): \quad f_{\alpha}^{(2)}=-\tau \partial_{t_{2}} f_{\alpha}^{(0)}+\tau^{2}\left(\partial_{t_{1}}+\boldsymbol{c}_{\alpha} \cdot \boldsymbol{\nabla}_{1}\right)^{2} f_{\alpha}^{(0)}=-\tau \partial_{t_{2}} f_{\alpha}^{(0)}-\tau\left(\partial_{t_{1}}+\boldsymbol{c}_{\alpha} \cdot \nabla_{1}\right) f_{\alpha}^{(1)}
\end{gathered}
$$

Consistently, this set of equations (up to $O\left(\epsilon^{2}\right)$ ) is identical to that obtained by applying the ChapmanEnskog expansion to the original discrete-velocity Boltzmann equation [2]. This set is sufficient to obtain the continuity equation and the Navier-Stokes equations by taking its statistical moments $\sum_{\alpha}(\cdot)$ and $\sum_{\alpha} \mathbf{c}_{\alpha}(\cdot)$, as briefly recalled in Appendix A. An essential point is that Eqs. (9), 110 and (11) come only from the first two terms of the recursive formulation, and that subsequent terms in the sum lead to higher orders in $\epsilon$. This is evidenced by reformulating Eq.(7) as

$$
f_{\alpha}=f_{\alpha}^{e q}+\sum_{m=1}^{\infty}(-\epsilon \tau)^{m}\left(\partial_{t_{1}}+\boldsymbol{c}_{\alpha} \cdot \nabla_{1}+\epsilon \partial_{t_{2}}+\cdots\right)^{m} f_{\alpha}^{e q}
$$


where the $m$-th term in the sum contributes at least to $O\left(\epsilon^{m}\right)$ in the multiple-scale expansion ${ }^{2}$. Therefore, it is enough to consider the first two terms to comply with the Navier-Stokes dynamics at the macroscopic level, i.e.

$$
f_{\alpha}=f_{\alpha}^{e q}-\tau\left(\partial_{t}+\boldsymbol{c}_{\alpha} \cdot \boldsymbol{\nabla}\right) f_{\alpha}^{e q}+\tau^{2}\left(\partial_{t}+\boldsymbol{c}_{\alpha} \cdot \boldsymbol{\nabla}\right)^{2} f_{\alpha}^{e q} .
$$

In the following, this second-order truncation will be the cornerstone of our numerical developments.

In practice, the LB scheme is obtained by integrating the discrete-velocity Boltzmann equation along the characteristics during a time interval $\delta_{t}$, and by approximating the integral of the collision term by a trapezium rule [2]. A convenient change of variables for the distribution functions is usually operated to render the scheme fully explicit. Namely,

$$
g_{\alpha}=f_{\alpha}+\frac{\delta_{t}}{2 \tau}\left(f_{\alpha}-f_{\alpha}^{e q}\right)
$$

where the $g_{\alpha}$ 's define a new set of distribution functions linked to the lattice discretization. This implies in particular that $g_{\alpha}^{e q}=f_{\alpha}^{e q}$ and $g_{\alpha}^{n e q} \equiv g_{\alpha}-g_{\alpha}^{e q}=\left(\tau_{g} / \tau\right) f_{\alpha}^{n e q}$ with the modified relaxation time

$$
\tau_{g}=\tau+\frac{\delta_{t}}{2} .
$$

In these new variables, the recursive formulation (restricted to second order) writes equivalently as

$$
g_{\alpha}(x, t)=g_{\alpha}^{e q}(x, t)-\tau_{g}\left(\partial_{t}+\boldsymbol{c}_{\alpha} \cdot \boldsymbol{\nabla}\right) g_{\alpha}^{e q}(x, t)+\tau_{g}\left(\tau_{g}-\frac{\delta_{t}}{2}\right)\left(\partial_{t}+\boldsymbol{c}_{\alpha} \cdot \boldsymbol{\nabla}\right)^{2} g_{\alpha}^{e q}(x, t) .
$$

In this article, the developments will be carried out with $f_{\alpha}$ and $\tau$ but could be easily transposed to $g_{\alpha}$ and $\tau_{g}$.

The final step towards a numerical scheme is the discretization of space and time variables. In this respect, Eq. 13] offers a large panel of possibilities. Since the equation implies particle derivatives, a natural choice (taken later in this study) is to integrate it along particle trajectories and, therefore, resort to an underlying uniform lattice structure, in the spirit of the standard lattice Boltzmann method. However, time and space variables can also be disentangled by treating independently the temporal and spatial derivatives, leading to so-called off-lattice Boltzmann schemes [13. These latter allow for grid cells with arbitrary shapes and thus provide a higher flexibility in the mesh geometry. Recent studies (starting from the DVBE) reveal the attractive potential of such schemes, which have now overcome the accuracy and stability issues initially encountered [14, 15, 16. The additional expense in computational costs is offset by the gain in the geometrical flexibility [17, 18. Combining several discretization methods is also possible to take advantage of the high efficiency (and low dissipation rate) of the stream-and-collide algorithm and the geometrical flexibility of off-lattice discretization for example, as recently demonstrated in [19, 20].

\subsection{Space-and-time discretization by backward finite differences}

At first, it would be tempting to recast Eq. (13) into a system of two coupled first-order differential equations reminiscent of the original discrete-velocity Boltzmann equation. Such system would write

$$
\begin{aligned}
\left(\partial_{t}+\boldsymbol{c}_{\alpha} \cdot \boldsymbol{\nabla}\right) f_{\alpha}^{e q} & =h_{\alpha} \\
\left(\partial_{t}+\boldsymbol{c}_{\alpha} \cdot \boldsymbol{\nabla}\right) h_{\alpha} & =\frac{1}{\tau^{2}}\left(f_{\alpha}-f_{\alpha}^{e q}\right)+\frac{1}{\tau} h_{\alpha} .
\end{aligned}
$$

Continuing the analogy, one could integrate the left-hand side along the characteristics and approximate the right-hand side by a trapezium rule. However, this would ultimately lead to a system of two coupled streamand-collide algorithms with more degrees of freedom (two sets of distribution functions) than the original

\footnotetext{
${ }^{2}$ Let us mention that $m=3$ and $m=4$ should be included in the sum to go beyond Navier-Stokes equations and recover the Burnett and super-Burnett equations, respectively.
} 
LB algorithm. Moreover, our preliminary simulations have shown that this double-distribution scheme is unstable.

It is rather proposed here to discretize the particle derivatives in Eq. (13) by backward finite-differences in time, namely

$$
\begin{aligned}
\left(\partial_{t}+\boldsymbol{c}_{\alpha} \cdot \nabla\right) f_{\alpha}^{e q[0]} & =\frac{1}{2 \delta_{t}}\left(3 f_{\alpha}^{e q[0]}-4 f_{\alpha}^{e q[-1]}+f_{\alpha}^{e q[-2]}\right)+O\left(\delta_{t}^{2}\right) \\
\left(\partial_{t}+\boldsymbol{c}_{\alpha} \cdot \nabla\right)^{2} f_{\alpha}^{e q[0]} & =\frac{1}{\delta_{t}^{2}}\left(f_{\alpha}^{e q[0]}-2 f_{\alpha}^{e q[-1]}+f_{\alpha}^{e q[-2]}\right)+O\left(\delta_{t}\right)
\end{aligned}
$$

where the short notation $f_{\alpha}^{e q[i]}$ stands for $f_{\alpha}^{e q}\left(\boldsymbol{x}+i \boldsymbol{c}_{\alpha} \delta_{t}, t+i \delta_{t}\right)$. This results in the formula

$$
f_{\alpha}^{n e q[0]}=\bar{\tau}\left[\left(\bar{\tau}-\frac{3}{2}\right) f_{\alpha}^{e q[0]}+2(1-\bar{\tau}) f_{\alpha}^{e q[-1]}+\left(\bar{\tau}-\frac{1}{2}\right) f_{\alpha}^{e q[-2]}\right] \quad \text { with } \bar{\tau}=\tau / \delta_{t} .
$$

This equation allows us to update the macroscopic variables $\rho(\boldsymbol{x}, t)$ and $\boldsymbol{u}(\boldsymbol{x}, t)$ from the values at neighbouring nodes at the previous time steps, by noting importantly that the non-equilibrium distributions $f_{\alpha}^{n e q[0]}$ must satisfy the solvability conditions ${ }^{3}$

$$
\sum_{\alpha} f_{\alpha}^{n e q[0]}=0 \quad \text { and } \quad \sum_{\alpha} f_{\alpha}^{n e q[0]} \boldsymbol{c}_{\alpha}=\mathbf{0}
$$

and that $\sum_{\alpha} f_{\alpha}^{e q[0]}=\rho(\boldsymbol{x}, t)$ and $\sum_{\alpha} f_{\alpha}^{e q[0]} \boldsymbol{c}_{\alpha}=(\rho \boldsymbol{u})(\boldsymbol{x}, t)$ by construction.

It is a priori desirable to have the same order of accuracy for the first and second differential operators. To do this, a four-point backward formula should be considered for the second-order operator

$$
\left(\partial_{t}+\boldsymbol{c}_{\alpha} \cdot \nabla\right)^{2} f_{\alpha}^{e q[0]}=\frac{1}{\delta_{t}^{2}}\left(2 f_{\alpha}^{e q[0]}-5 f_{\alpha}^{e q[-1]}+4 f_{\alpha}^{e q[-2]}-f_{\alpha}^{e q[-3]}\right)+O\left(\delta_{t}^{2}\right)
$$

which this time yields

$$
f_{\alpha}^{n e q[0]}=\bar{\tau}\left[\left(2 \bar{\tau}-\frac{3}{2}\right) f_{\alpha}^{e q[0]}+(2-5 \bar{\tau}) f_{\alpha}^{e q[-1]}+\left(4 \bar{\tau}-\frac{1}{2}\right) f_{\alpha}^{e q[-2]}-\bar{\tau} f_{\alpha}^{e q[-3]}\right] .
$$

In order to bridge the two discretizations (21) and (24), it is here proposed to leave the weight related to $f_{\alpha}^{e q[-3]}$ as a free parameter $\gamma$. After manipulating Taylor expansions, this leads to the parametric family of finite-difference schemes

$$
f_{\alpha}^{n e q[0]}=\bar{\tau}\left(\left(-\gamma+\bar{\tau}-\frac{3}{2}\right) f_{\alpha}^{e q[0]}+(3 \gamma+2(1-\bar{\tau})) f_{\alpha}^{e q[-1]}+\left(-3 \gamma+\bar{\tau}-\frac{1}{2}\right) f_{\alpha}^{e q[-2]}+\gamma f_{\alpha}^{e q[-3]}\right)
$$

consistent with the original differential equation $(13)$. One must mention that the sum of the coefficients in Eq. 25) being zero ensures that this scheme is mass-conserving. Eq. (25) also shares some similarity with the multi-step LB methods 21, in which the distribution functions are expressed according to their value at previous times and neighbouring nodes. If $\gamma=0$, the first and second differential operators are approximated respectively at second and first order and Eq. 21) is obtained. If $\gamma=-\bar{\tau}$, the first and second derivatives are approximated with a second-order accuracy and Eq. 24 is obtained. If $\gamma=-\bar{\tau}+\frac{1}{3}$, the first and second derivatives are approximated respectively with a third-order and second-order accuracy. Interestingly, we will see later that the parameter $\gamma$ can be optimized to significantly reduce the discretization errors.

At this point, we can mention that the regularized LB method introduced by Latt \& Chopard 22$]$ resembles our modeling in that both methods conform to the Navier-Stokes equations at the macroscopic level, and provide a reconstruction of the non-equilibrium component of the distributions by an alternative

\footnotetext{
${ }^{3}$ The BGK collision operator conserves microscopic mass and momentum.
} 
means to the standard LB scheme. However, the regularized LB is a local reconstruction (in $(\mathbf{x}, t))$ that can be viewed as a projection on a restricted basis of Hermite polynomials, while our method is a nonlocal reconstruction based on the equilibrium distributions. If the source of numerical dissipation may be attributed to the projection operation (in the velocity space) in the regularized method, it will be rather related to the spatio-temporal discretization in our case. Concerning the link-wise artificial compressibility (LW-AC) method, it shares with the present modeling its finite-difference formulation. However, the asymptotic analysis of this model (developed in appendix B in [6]) does not yield the high-order temporal and mixed derivatives that we obtain through the recursion. Therefore, the two methods cannot infer from each other. We suspect that the "empirical" substitution of the distributions by their equilibrium components (Eqs. $(5,10)$ in $[6])$ is the main factor behind this difference.

From Eq. 25) and by resorting to the solvability conditions 22, the flow variables are updated according to the sums

$$
\begin{aligned}
\rho(\boldsymbol{x}, t) & =\sum_{\alpha} f_{\alpha}^{*}, \\
(\rho \boldsymbol{u})(\boldsymbol{x}, t) & =\sum_{\alpha} f_{\alpha}^{*} \boldsymbol{c}_{\alpha}
\end{aligned}
$$

with the effective distributions $f_{\alpha}^{*}$ given by

$$
f_{\alpha}^{*}=\frac{3 \gamma+2(1-\bar{\tau})}{\gamma-\bar{\tau}+\frac{3}{2}} f_{\alpha}^{e q[-1]}+\frac{-3 \gamma+\bar{\tau}-\frac{1}{2}}{\gamma-\bar{\tau}+\frac{3}{2}} f_{\alpha}^{e q[-2]}+\frac{\gamma}{\gamma-\bar{\tau}+\frac{3}{2}} f_{\alpha}^{e q[-3]}
$$

Since, $f_{\alpha}^{e q}$ depends on flow variables only (see Eq. (5) $), f_{\alpha}^{*}$ can be evaluated from the previous values of $\rho$ and $\boldsymbol{u}$ at neighbouring lattice nodes and previous time steps. It can be pointed out that the schemes not only advance in time the density and the velocity fields but also provide a direct estimation of the rate-ofstrain tensor through the second-order non-equilibrium moment: $-2 \rho \tau c_{s}^{2} \boldsymbol{S} \approx \sum_{\alpha} f_{\alpha}^{n e q} \boldsymbol{c}_{\alpha} \boldsymbol{c}_{\alpha}$. The first steps of the algorithm can be performed by using the standard (one-step) lattice Boltzmann scheme. The A-B memory access pattern, commonly referred to as "ping-pong buffering", is typically used in the LB scheme for better performance 2]. It requires two sets of distributions at each node, and thus 18 and 38 values for the D2Q9 and D3Q19 lattices. These two numbers are reduced to 12 and 16 in the present modelling (with $\gamma \neq 0$ ). In terms of computations, our algorithm requires to upload the three previous values of $\rho$ and $\mathbf{u}$ at neighbouring positions to reconstruct $f_{\alpha}^{e q[-1]}, f_{\alpha}^{e q[-2]}$ and $f_{\alpha}^{e q[-3]}$ and evaluate $f_{\alpha}^{*}$ for all directions $\alpha$. The updated values of $\rho$ and $\rho \boldsymbol{u}$ are eventually computed as the statistical moments of $f_{\alpha}^{*}$. A schematic view of the algorithm is given in Fig. 2.

$$
\left\{\begin{array}{l}
\rho^{[-1]}, \rho^{[-2]}, \rho^{[-3]} \\
\boldsymbol{u}^{[-1]}, \boldsymbol{u}^{[-2]}, \boldsymbol{u}^{[-3]}
\end{array} \underset{\text { Eq.(5) }}{\longrightarrow} f_{\alpha}^{e q[-1]}, f_{\alpha}^{e q[-2]}, f_{\alpha}^{e q[-3]} \underset{\text { Eq.(28) }}{\longrightarrow} f_{\alpha}^{*} \longrightarrow\left\{\begin{array}{l}
\rho^{[0]}=\sum_{\alpha} f_{\alpha}^{*} \\
\rho \boldsymbol{u}^{[0]}=\sum_{\alpha} f_{\alpha}^{*} \boldsymbol{c}_{\alpha}
\end{array}\right.\right.
$$

Figure 2: Schematic view of the algorithm.

In the following, our scheme will be referred to as recursive finite-difference lattice Boltzmann scheme with reference to the recursive formulation of the original DVBE.

\subsection{Simplified prediction-correction Lattice Boltzmann algorithm}

Interestingly, a so-called simplified LB method relying solely on the equilibrium distributions has recently been introduced by Chen et al. in [7] and has proven to be relevant for many applications [23, 24, 25. In the following, we shall see that this method may be recast in the present framework by resorting to different levels of approximation in the recursive formulation of the discrete-velocity Boltzmann equation. 
The simplified LB method proceeds in a two-step prediction-correction procedure. The density and the fluid momentum are first predicted as

$$
\begin{aligned}
\rho^{*}(\boldsymbol{x}, t) & =\sum_{\alpha} f_{\alpha}^{e q[-1]}, \\
(\rho \boldsymbol{u})^{*}(\boldsymbol{x}, t) & =\sum_{\alpha} \boldsymbol{c}_{\alpha} f_{\alpha}^{e q[-1]}
\end{aligned}
$$

which is equivalent to considering a recursive expansion restricted to $m=1$ and a first-order backward finite-difference approximation of the derivative, i.e.

$$
f_{\alpha}^{n e q[0]} \simeq-\bar{\tau}\left(f_{\alpha}^{e q[0]}-f_{\alpha}^{e q[-1]}\right) .
$$

Obviously, this dynamics does not conform to the Navier-Stokes equations and must be corrected.

A Taylor expansion in time of $f_{\alpha}^{e q[-1]} \equiv f_{\alpha}^{e q}\left(\boldsymbol{x}-\boldsymbol{c}_{\alpha} \delta_{t}, t-\delta_{t}\right)$ gives

$$
f_{\alpha}^{e q[-1]}=f_{\alpha}^{e q[0]}-\delta_{t}\left(\partial_{t}+\boldsymbol{c}_{\alpha} \cdot \boldsymbol{\nabla}\right) f_{\alpha}^{e q[0]}+\frac{\delta_{t}^{2}}{2}\left(\partial_{t}+\boldsymbol{c}_{\alpha} \cdot \boldsymbol{\nabla}\right)^{2} f_{\alpha}^{e q[0]}+o\left(\delta_{t}{ }^{2}\right),
$$

which may be reformulated as

$$
f_{\alpha}^{e q[-1]}=f_{\alpha}^{e q[0]}-\delta_{t}\left(1-\frac{1}{2 \bar{\tau}}\right)\left(\partial_{t}+\boldsymbol{c}_{\alpha} \cdot \boldsymbol{\nabla}\right) f_{\alpha}^{e q[0]}+\frac{\delta_{t}^{2}}{2 \bar{\tau}^{2}} f_{\alpha}^{n e q[0]}+o\left(\delta_{t}^{2}\right)
$$

by expressing the second-order derivative from the recursive expansion 13 . The first and second moments then yield at $(\boldsymbol{x}, t)$

$$
\begin{gathered}
\rho^{*}=\rho-\delta_{t}\left(1-\frac{1}{2 \bar{\tau}}\right)\left(\partial_{t} \rho+\nabla \cdot(\rho \boldsymbol{u})\right)+o\left(\delta_{t}{ }^{2}\right), \\
(\rho \boldsymbol{u})^{*}=\rho \boldsymbol{u}-\delta_{t}\left(1-\frac{1}{2 \bar{\tau}}\right)\left(\partial_{t}(\rho \boldsymbol{u})+\boldsymbol{\nabla} \cdot \boldsymbol{\Pi}^{e q}\right)+o\left(\delta_{t}{ }^{2}\right) .
\end{gathered}
$$

Therefore, no correction is needed for the density, i.e. $\rho(\boldsymbol{x}, t)=\rho^{*}(\boldsymbol{x}, t)$, to comply with the continuity equation, whereas the momentum must be corrected as

$$
\rho \boldsymbol{u}=(\rho \boldsymbol{u})^{*}-\delta_{t}\left(1-\frac{1}{2 \bar{\tau}}\right) \boldsymbol{\nabla} \cdot \boldsymbol{\Pi}^{n e q}+o\left(\delta_{t}{ }^{2}\right) \quad \text { with } \quad \boldsymbol{\Pi}^{n e q}=\sum_{\alpha} f_{\alpha}^{n e q} \boldsymbol{c}_{\alpha} \boldsymbol{c}_{\alpha}
$$

to be consistent with the Navier-Stokes dynamics. The divergence term needs to be discretized to complete the model. To do so, a Taylor expansion in space of $f_{\alpha}^{n e q}(\boldsymbol{x}, t)$ gives

$$
f_{\alpha}^{n e q}\left(\boldsymbol{x} \pm \frac{1}{2} \boldsymbol{c}_{\alpha} \delta_{t}, t\right)=f_{\alpha}^{n e q}(\boldsymbol{x}, t) \pm \frac{1}{2} \delta_{t}\left(\boldsymbol{c}_{\alpha} \cdot \boldsymbol{\nabla}\right) f_{\alpha}^{n e q}(\boldsymbol{x}, t)+\frac{1}{4} \delta_{t}^{2}\left(\boldsymbol{c}_{\alpha} \cdot \boldsymbol{\nabla}\right)^{2} f_{\alpha}^{n e q}(\boldsymbol{x}, t)+o\left(\delta_{t}^{2}\right)
$$

which allows us to finally rewrite the correction for the momentum as

$$
(\rho \boldsymbol{u})(\boldsymbol{x}, t)=(\rho \boldsymbol{u})^{*}(\boldsymbol{x}, t)-\left(1-\frac{1}{2 \bar{\tau}}\right) \sum_{\alpha} \boldsymbol{c}_{\alpha}\left(f_{\alpha}^{n e q}\left(\boldsymbol{x}+\frac{1}{2} \boldsymbol{c}_{\alpha} \delta_{t}, t\right)-f_{\alpha}^{n e q}\left(\boldsymbol{x}-\frac{1}{2} \boldsymbol{c}_{\alpha} \delta_{t}, t\right)\right)+o\left(\delta_{t}{ }^{2}\right) .
$$

The approach is so far general and results from manipulating the discrete-velocity Boltzmann equation and using Taylor expansions at second order. Interestingly, Chen et al. 7] then proposed to simplify Eq. (38) by evaluating the correction at intermediate time $t-\delta t / 2$ and by approximating the non-equilibrium distributions as

$$
\begin{aligned}
& f_{\alpha}^{n e q}\left(\boldsymbol{x}+\frac{1}{2} \boldsymbol{c}_{\alpha} \delta_{t}, t-\frac{\delta_{t}}{2}\right) \simeq-\bar{\tau}\left(f_{\alpha}^{e q *}\left(\boldsymbol{x}+\boldsymbol{c}_{\alpha} \delta_{t}, t\right)-f_{\alpha}^{e q}\left(\boldsymbol{x}, t-\delta_{t}\right)\right) \\
& f_{\alpha}^{n e q}\left(\boldsymbol{x}-\frac{1}{2} \boldsymbol{c}_{\alpha} \delta_{t}, t-\frac{\delta_{t}}{2}\right) \simeq-\bar{\tau}\left(f_{\alpha}^{e q *}(\boldsymbol{x}, t)-f_{\alpha}^{e q}\left(\boldsymbol{x}-\boldsymbol{c}_{\alpha} \delta_{t}, t-\delta_{t}\right)\right) .
\end{aligned}
$$


This is equivalent to considering a recursive expansion restricted to $m=1$ and a second-order central finitedifference approximation of the particle derivative. As expected in a prediction-correction procedure, the distributions at time $t$ are evaluated from the predicted density and momentum, i.e. $f_{\alpha}^{e q *}=f_{\alpha}^{e q}\left(\rho^{*}, \boldsymbol{u}^{*}\right)$. The resulting algorithm is indeed simple and may be summarized as

- prediction step:

$$
\begin{aligned}
\rho^{*}(\boldsymbol{x}, t) & =\sum_{\alpha} f_{\alpha}^{e q[-1]} \\
(\rho \boldsymbol{u})^{*}(\boldsymbol{x}, t) & =\sum_{\alpha} \boldsymbol{c}_{\alpha} f_{\alpha}^{e q[-1]}
\end{aligned}
$$

- correction step:

$$
\begin{aligned}
\rho(\boldsymbol{x}, t) & =\rho^{*}(\boldsymbol{x}, t) \\
(\rho \boldsymbol{u})(\boldsymbol{x}, t) & =(\rho \boldsymbol{u})^{*}(\boldsymbol{x}, t)-\left(\bar{\tau}-\frac{1}{2}\right)\left[(\rho \boldsymbol{u})\left(\boldsymbol{x}, t-\delta_{t}\right)-\sum_{\alpha} \boldsymbol{c}_{\alpha} f_{\alpha}^{e q}\left(\rho^{*}, \boldsymbol{u}^{*}\right)\left(\boldsymbol{x}+\boldsymbol{c}_{\alpha} \delta_{t}, t\right)\right]
\end{aligned}
$$

Therefore, this simplified $L B$ scheme can be recast in the present modeling framework (under specific assumptions). It will be considered for comparisons in the following numerical tests.

\section{Numerical simulations}

In this section, two flow configurations are considered to assess the validity of our recursive finitedifference scheme (abbreviated as RFD, Eqs. (26), (27), and (28)) against the prediction-correction scheme (PRECORR, Eqs. (41), 42), (43), and (44)) and the standard stream-and-collide LB algorithm (LBM, Eq. (45)). This latter expresses as

$$
g_{\alpha}\left(\boldsymbol{x}+\boldsymbol{c}_{\alpha} \delta_{t}, t+\delta_{t}\right)=g_{\alpha}(\boldsymbol{x}, t)-\frac{1}{\bar{\tau}_{g}}\left(g_{\alpha}(\boldsymbol{x}, t)-g_{\alpha}^{e q}(\boldsymbol{x}, t)\right)
$$

with $\bar{\tau}_{g}=\bar{\tau}+1 / 2$ and $g_{\alpha}^{e q}=f_{\alpha}^{e q}$. The RFD algorithm is initialized by performing the first two steps with the standard LB method. Two different values of the adjustable parameter $\gamma$ are considered in the analysis, namely $\gamma=0$ and an optimal value obtained by a trial-and-error procedure (detailed later). To improve the stability of the LB scheme, the equilibrium distributions have been developed at fourth order in Hermite expansion in the various schemes [26, 27], i.e.

$$
\begin{aligned}
f_{\alpha}^{e q}(\rho, \boldsymbol{u}) & =w_{\alpha} \rho\left[1+\frac{\boldsymbol{c}_{\alpha} \cdot \boldsymbol{u}}{c_{s}{ }^{2}}+\frac{\left(\boldsymbol{c}_{\alpha} \cdot \boldsymbol{u}\right)^{2}}{2 c_{s}{ }^{4}}-\frac{\boldsymbol{u}^{2}}{2 c_{s}{ }^{2}}\right. \\
& \left.+\frac{\left(\boldsymbol{c}_{\alpha} \cdot \boldsymbol{u}\right)^{3}}{6 c_{s}{ }^{6}}-\frac{\left(\boldsymbol{c}_{\alpha} \cdot \boldsymbol{u}\right) \boldsymbol{u}^{2}}{2 c_{s}{ }^{4}}+\frac{\left(\boldsymbol{c}_{\alpha} \cdot \boldsymbol{u}\right)^{4}}{24 c_{s}{ }^{8}}-\frac{\left(\boldsymbol{c}_{\alpha} \cdot \boldsymbol{u}\right)^{2} \boldsymbol{u}^{2}}{4 c_{s}{ }^{6}}+\frac{\boldsymbol{u}^{4}}{8 c_{s}{ }^{4}}\right]
\end{aligned}
$$

In the remainder of the article, all quantities are expressed in lattice units obtained by normalizing space and time by the lattice spacing and the time step, respectively.

\subsection{Double shear-layer flow}

The double shear-layer flow is a classical test case for numerical methods. Two thin horizontal shear layers are located at $y=L / 4$ and $y=3 L / 4$ in a two-dimensional periodic domain of size $[0, L] \times[0, L]$. A small vertical velocity perturbation is added in order to disturb the flow. A Kelvin-Helmholtz instability is expected to develop with two counter-rotating growing vortices. The thinning of the shear layers can lead to the development of spurious vortices and possibly the blow-up of the simulation. 
The initial velocity field is

$$
\begin{array}{ll}
u_{x}(\boldsymbol{x}, 0)=U_{0} \tanh \left(k\left(\frac{y}{L}-\frac{1}{4}\right)\right) & \text { for } \quad y \leq L / 2 \\
u_{x}(\boldsymbol{x}, 0)=U_{0} \tanh \left(k\left(\frac{3}{4}-\frac{y}{L}\right)\right) & \text { for } \quad y \geq L / 2 \\
u_{y}(\boldsymbol{x}, 0)=U_{0} \delta \sin \left(2 \pi\left(\frac{x}{L}+\frac{1}{4}\right)\right) &
\end{array}
$$

where $U_{0}$ is the characteristic velocity of the flow, $k / L$ is inversely proportional to the thickness of the two shear layers and $\delta$ is the amplitude coefficient of the initial perturbation in velocity. Following previous works, the double shear-layer flow is simulated with $k=80$ and $\delta=5 \%$ [28, 29]. The reference velocity $U_{0}$ is fixed so that the Mach number is $\mathrm{Ma}=U_{0} / c_{s}=\sqrt{3} U_{0}=0.3$. The length $L$ corresponds to the resolution of the simulation (in lattice units) and is considered as a parameter. The Reynolds number is kept constant at $\operatorname{Re}=U_{0} L / \nu=3 \cdot 10^{4}$ by adjusting the viscosity. This configuration corresponds to a challenging test case that can become unstable at low resolutions. Finally, let us mention that the initial density (or pressure) field is not known. To circumvent this difficulty, the iterative procedure proposed by Mei et al. [30] was used to generate the distribution functions in a consistent manner and avoid transient errors; the initial density was set to unity and the stopping error criterion was taken to be $10^{-8}$ in the iterative procedure.

In Fig. 3 the vorticity for various resolutions is displayed at the same convective time $t^{*}=1$ with $t^{*} \equiv t U_{0} / L$ for the different algorithms. For the RFD algorithm, the top shear layer refers to a simulation with $\gamma=0$, whereas the bottom shear layer is simulated with an optimized $\gamma \neq 0$ obtained by a trial-anderror method. An analytical approach (based on a von-Neumann analysis) will be introduced later in the article to infer the optimal $\gamma$. For the moment, the optimal $\gamma$ is found by incremental testing, i.e. by trying successive values of $\gamma$ with an increment of 0.01 . The optimal $\gamma$ minimizes the error made on the averaged kinetic energy with respect to a reference solution in the interval $0 \leq t^{*} \leq 2$. In practice, we used the solution of the LBM simulation at the highest resolution $(L=512)$ as reference.

At low resolution $(L=128)$ the LBM simulation is unstable whereas the RFD and PRECORR simulations remain stable but the counter-rotating vortices are obviously over-damped. At intermediate resolution ( $L=256)$ the LBM gains in stability but spurious vortices still appear where the shear layer is the thinnest. These vortices are not observed in the RFD and PRECORR simulations, however, the latter produce a larger numerical dissipation as evidenced by the diffusion of the shear layer. Interestingly, the situation can be greatly improved by selecting the optimal parameter $\gamma=0.18$ in the RFD algorithm. Finally, all schemes give satisfactory results and converge towards the same solution at high resolution $(L=512)$ except for the RFD with $\gamma=0$, for which the vortex is still too dissipated.

In summary, these first qualitative results show that the RFD and PRECORR algorithms allow us to simulate the double-shear-layer flow (at all resolutions) but this comes with an accentuated numerical dissipation as compared to the original LB scheme. Nevertheless, it is possible to reduce significantly the numerical dissipation in the RFD simulation by adjusting the value of the parameter $\gamma$. This is quantitatively confirmed in Fig. 4, where the mean kinetic energy is plotted at the intermediate resolution for the different algorithms. At this resolution, the RFD simulation with the optimized $\gamma$ is stable and very close to the reference LBM solution.

\subsection{Taylor-Green vortex flow}

The two-dimensional Taylor-Green vortex flow is another meaningful test case as it provides an exact analytical solution for the Navier-Stokes equations (at low Reynolds number). The solution reads for a fully 

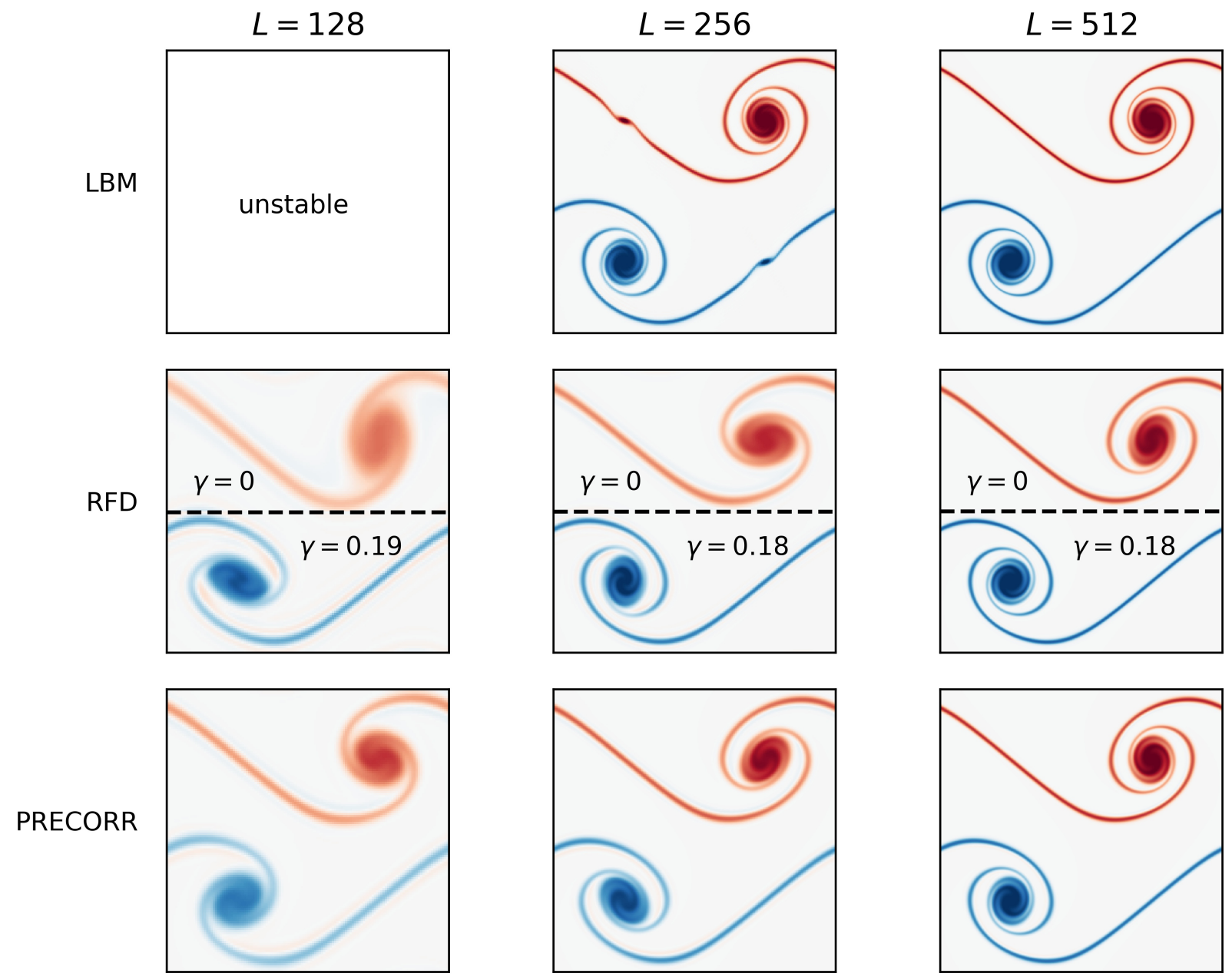

Figure 3: Double shear-layer simulations. Normalized vorticity at convective time $t^{*} \equiv t U_{0} / L=1$ for resolutions $L=128$, $L=256$ and $L=512$. The LBM simulation is unstable at $L=128$.

periodic domain of size $[0, L] \times[0, L]$

$$
\begin{aligned}
u_{x}(\boldsymbol{x}, t) & =-U_{0} \sqrt{\frac{k_{y}}{k_{x}}} \cos \left(k_{x} x\right) \sin \left(k_{y} y\right) \exp \left(-t / T_{\nu}\right) \\
u_{y}(\boldsymbol{x}, t) & =U_{0} \sqrt{\frac{k_{x}}{k_{y}}} \sin \left(k_{x} x\right) \cos \left(k_{y} y\right) \exp \left(-t / T_{\nu}\right) \\
\rho(\boldsymbol{x}, t) & =1-\frac{U_{0}^{2}}{4 c_{s}^{2}}\left(\frac{k_{y}}{k_{x}} \cos \left(2 k_{x} x\right)+\frac{k_{x}}{k_{y}} \cos \left(2 k_{y} y\right)\right) \exp \left(-2 t / T_{\nu}\right)
\end{aligned}
$$

where $U_{0}$ is the initial characteristic velocity, $k_{x}=k_{y}=2 \pi / L$, and $T_{\nu}=1 /\left[\left(k_{x}^{2}+k_{y}^{2}\right) \nu\right]$ is the characteristic decay time. The initial distribution functions for the LBM algorithm are taken at equilibrium value, i.e. $f_{\alpha}(\boldsymbol{x}, 0)=f_{\alpha}^{e q}(\rho(\boldsymbol{x}, 0), \boldsymbol{u}(\boldsymbol{x}, 0))$; here both $\rho(\boldsymbol{x}, 0)$ and $\boldsymbol{u}(\boldsymbol{x}, 0)$ are known. At low Reynolds number, the initial vortices decay in time with an exponential rate $T_{\nu}$ due to the viscous dissipation. As before, various simulations are performed with the RFD, PRECORR and LBM schemes and compared with the analytical 


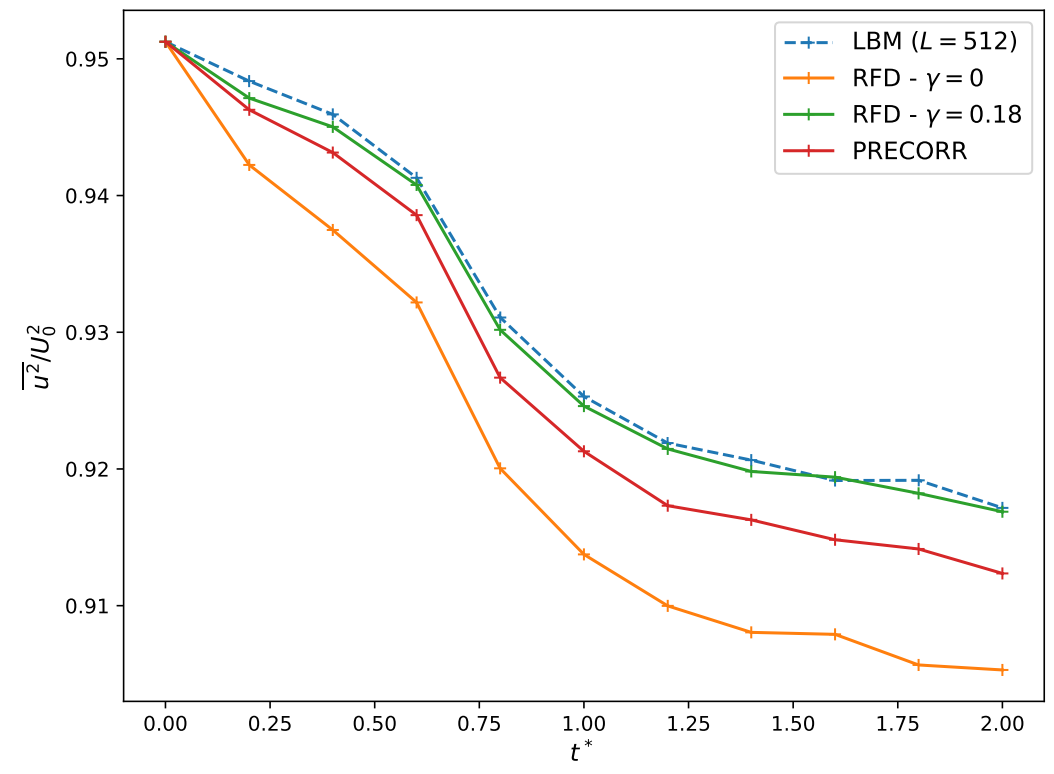

Figure 4: Double shear-layer simulations. Normalized averaged kinetic energy according to the convective time $t^{*}$ for the intermediate resolution $L=256$. The reference simulation refers to the LBM simulation at high resolution $L=512$.

solution. The relative error is quantified by using the $L^{2}$-norm over the entire computational domain, i.e.

$$
\varepsilon_{\varphi}\left(t^{*}\right)=\sqrt{\frac{\sum_{\boldsymbol{x}}\left(\varphi_{\text {simulated }}\left(\boldsymbol{x}, t^{*}\right)-\varphi_{\text {analytical }}\left(\boldsymbol{x}, t^{*}\right)\right)^{2}}{\sum_{\boldsymbol{x}} \varphi_{\text {analytical }}^{2}\left(\boldsymbol{x}, t^{*}\right)}}
$$

for the variables $\varphi=\rho, u_{x}$ and $u_{y}$ at the normalized diffusive time $t^{*}=t / T_{\nu}$. In practice, only the error in $\varepsilon_{u_{x}}$ will be examined, the error $\varepsilon_{u_{y}}$ being of the same order of magnitude. Table 1 gathers the parameters of the different simulations and the related figures.

\begin{tabular}{|c|c|c|c|}
\hline & $L$ & $\nu=\bar{\tau} / 3$ & Re \\
\hline Fig. 5 & variable & 0.01 & 100 \\
\hline Fig. $\overline{\overline{6}}$ & 32 & variable & 100 \\
\hline Fig. 7 & 32 & 0.01 & variable \\
\hline Fig. $\overline{8}$ & 32 & 0.01 & 100 \\
\hline
\end{tabular}

Table 1: Independent parameters of the performed simulations. The characteristic velocity $U_{0}$ is given by $U_{0}=\operatorname{Re} \nu / L$ and the Mach number is $\mathrm{Ma}=\sqrt{3} U_{0}$.

At first, different domain sizes (or resolutions) are considered, namely $L=32,64,128$ and 256, and a convergence analysis is carried out. Here, it is appropriate to adopt a diffusive scaling, meaning that $\delta_{x}{ }^{2} / \delta_{t}$ is kept constant through all simulations. Consequently, the relaxation time (or the viscosity) is constant in lattice units but the Mach number varies inversely to the resolution. The similitude in the Mach number can be disregarded as long as acoustic features are ignored, i.e. the Mach number remains low. Such condition is met here. The characteristic velocity is given by

$$
U_{0}=\frac{\operatorname{Re} \nu}{L} .
$$

The Taylor-Green vortex flow is simulated up to two decay times $\left(t^{*}=2\right)$ at $\operatorname{Re}=100$ with $\nu=0.01$. The Mach number varies as $\mathrm{Ma}=\sqrt{3} / L \ll 1$ with the resolution $L$. The relative errors are evaluated at 
$t^{*}=2$ and shown in Fig. 5. For all schemes, a fourth and second-order convergence are found for the density and the velocity respectively. This is consistent with the second-order accuracy in space of the different algorithms. The difference of order between the density and the velocity agrees with the hypothesis that typically $\delta \rho \sim \rho_{0} u^{2} / c_{s}^{2}$ in the weak-compressibility limit. Nevertheless, while sharing the same order of convergence, the initial error for the RFD algorithm with $\gamma=0$ and the PRECORR algorithm is much greater. The optimal value $\gamma=0.15$ in the RFD algorithm reduces this initial error at a level close to that of the LBM simulation. No variation of the optimal $\gamma$ was found for the different resolutions with a constant relaxation time.

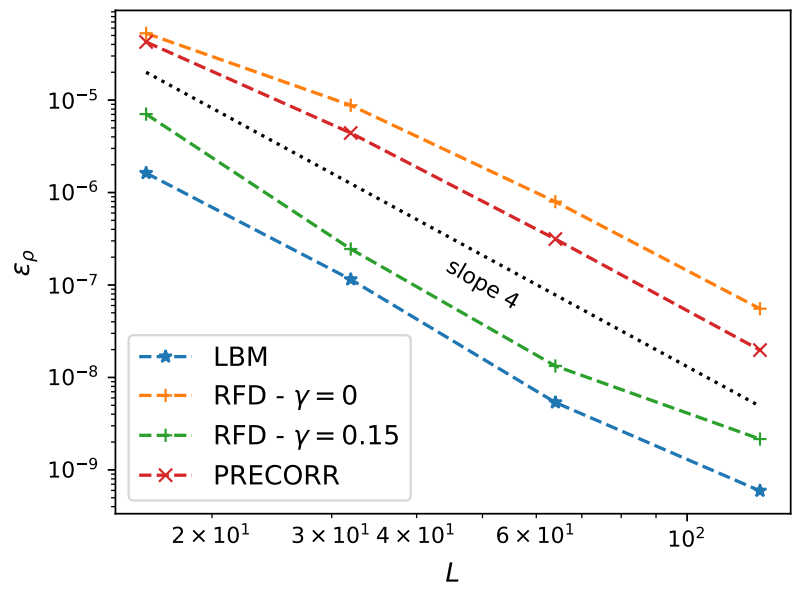

(a) $\varepsilon_{\rho}$

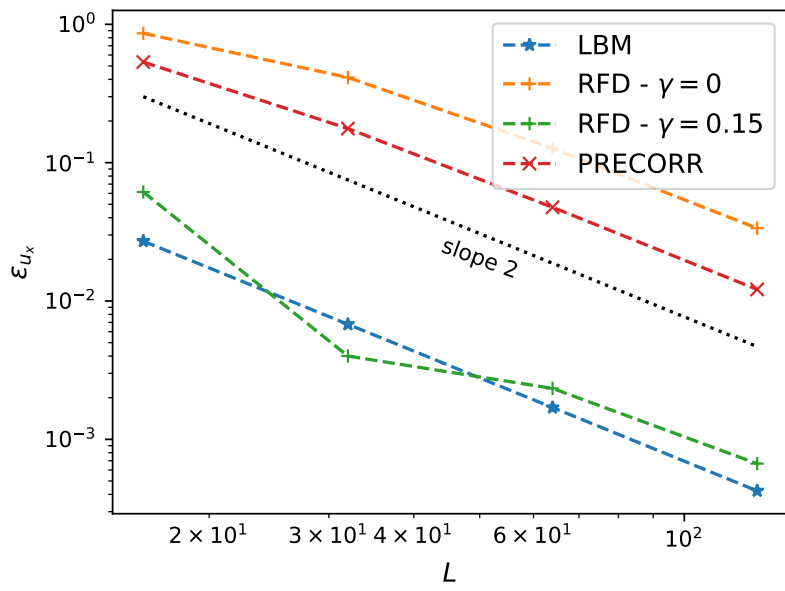

(b) $\varepsilon_{u_{x}}$

Figure 5: Taylor-Green vortex flow simulations. $L^{2}$-norm (relative) error at time $t=2 T_{\nu}$ for the different resolutions $L=16$, $L=32, L=64$ and $L=128$.

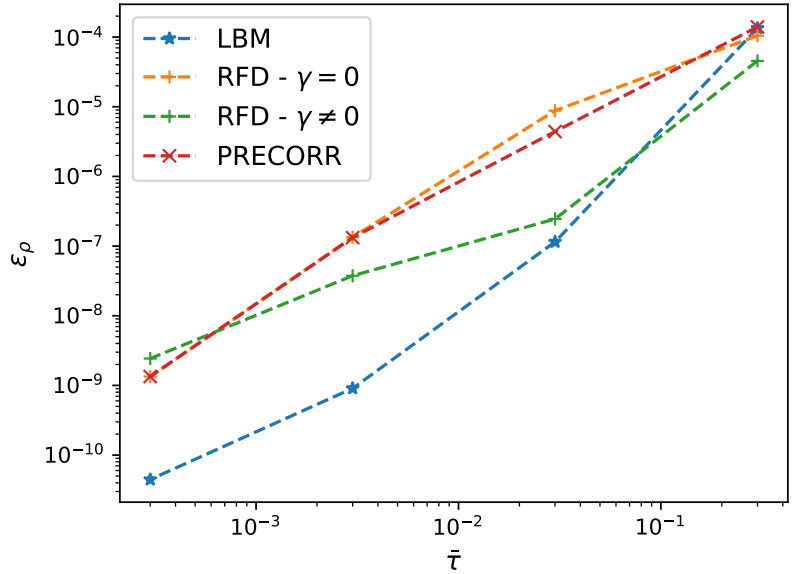

(a) $\varepsilon_{\rho}$

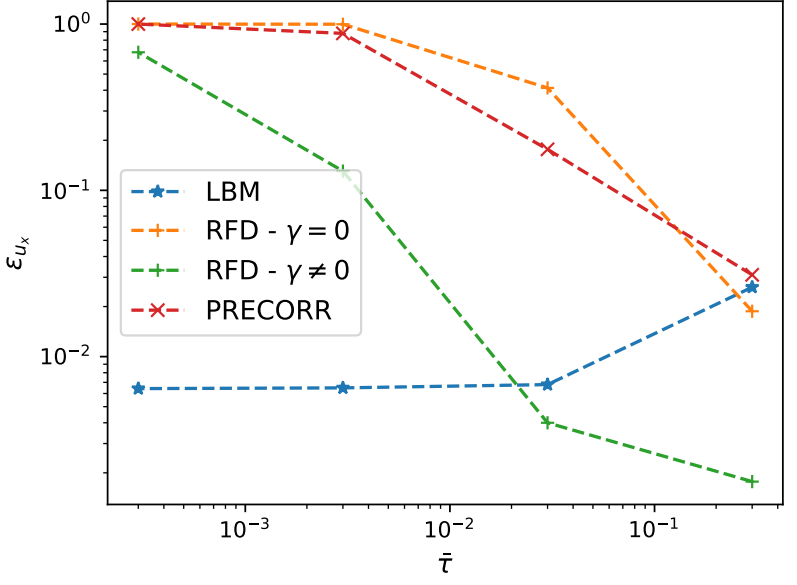

(b) $\varepsilon_{u_{x}}$

Figure 6: Taylor-Green vortex flow simulations. $L^{2}$-norm (relative) error at time $t=2 T_{\nu}$ for various relaxation times, $\bar{\tau}=3 \nu$ in lattice units. The optimal $\gamma$ in the RFD algorithm is $\gamma=0.17$ for $\bar{\tau}=0.0003$ and $\bar{\tau}=0.003, \gamma=0.15$ for $\bar{\tau}=0.03$ and $\gamma=-0.07$ for $\bar{\tau}=0.3$

The influence of the relaxation time, or viscosity, at constant Reynolds number $\mathrm{Re}=100$ and resolution $L=32$ is now examined. Changing the relaxation time $(\bar{\tau}=3 \nu$ in lattice units $)$ leads to a variable Mach number with $\mathrm{Ma}=(\operatorname{Re} / \sqrt{3} L) \bar{\tau}$. It is observed in Fig 6a that the error in density naturally increases with 
$\bar{\tau}$. Large errors on the velocity are observed in Fig. 6b for small relaxation times as compared to the LBM algorithm. The numerical dissipation of the RFD algorithm with $\gamma=0$ and the PRECORR algorithm damages the numerical accuracy at this low resolution $(L=32)$. At the smallest viscosity, the numerical dissipation inherent to the finite-difference discretization exceeds the actual dissipation and the optimization of $\gamma$ no longer helps to reduce $\varepsilon_{u_{x}}$ in the RFD algorithm. This obviously points to an obvious limitation of this type of approach in the limit of vanishing viscosity, unless the resolution is considerably increased. On the other hand, at high viscosity the RFD algorithm with an optimized $\gamma$ appears to provide the most accurate value for the velocity, the LBM solution being degraded by errors in Mach number. Such errors, which are $a$ priori also present in the finite-difference approach, can be "compensated" by picking the optimal $\gamma$. Finally, let us notice that the optimal $\gamma$ depends on $\bar{\tau}$.

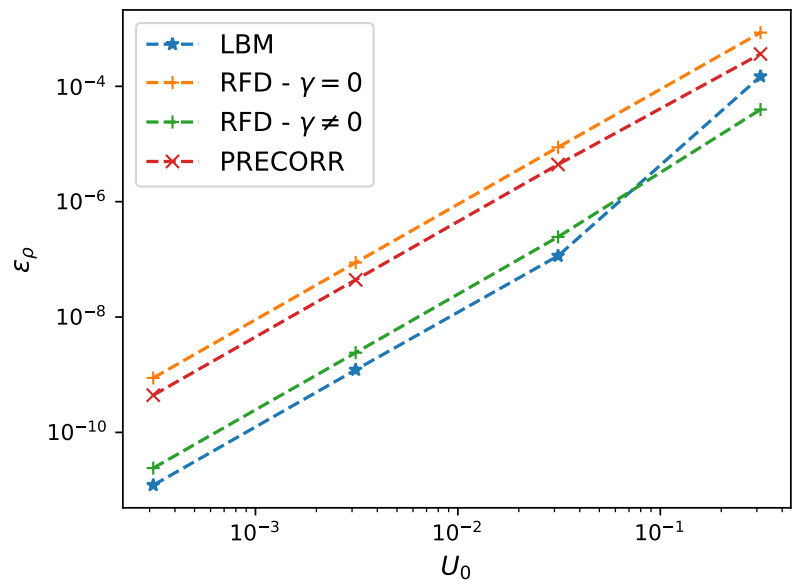

(a) $\varepsilon_{\rho}$

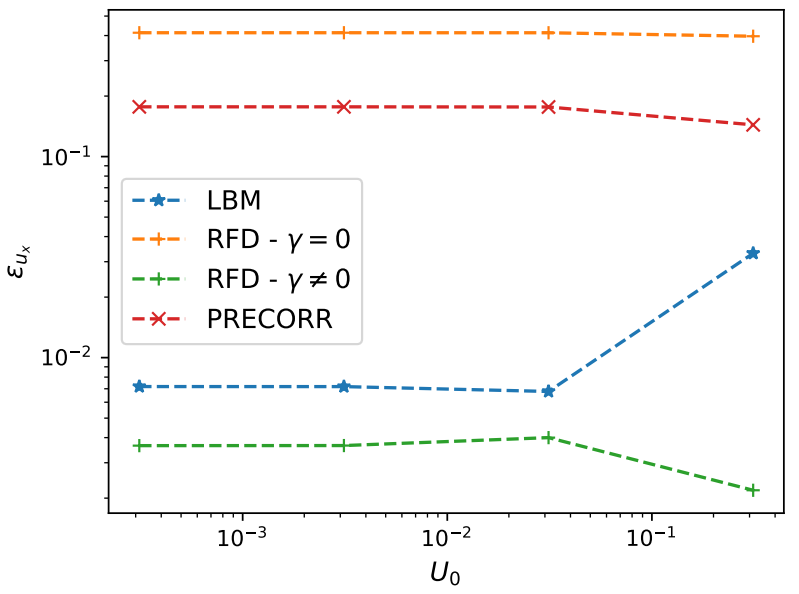

(b) $\varepsilon_{u_{x}}$

Figure 7: Taylor-Green vortex flow simulations. $L^{2}$-norm (relative) error at time $t=2 T_{\nu}$. The optimal $\gamma$ in the RFD simulation is $\gamma=0.15$ for $U_{0}=3.125 \cdot 10^{-4}, 3.125 \cdot 10^{-3}, 3.125 \cdot 10^{-2}$ and $\gamma=0.14$ for $U_{0}=0.3125$.

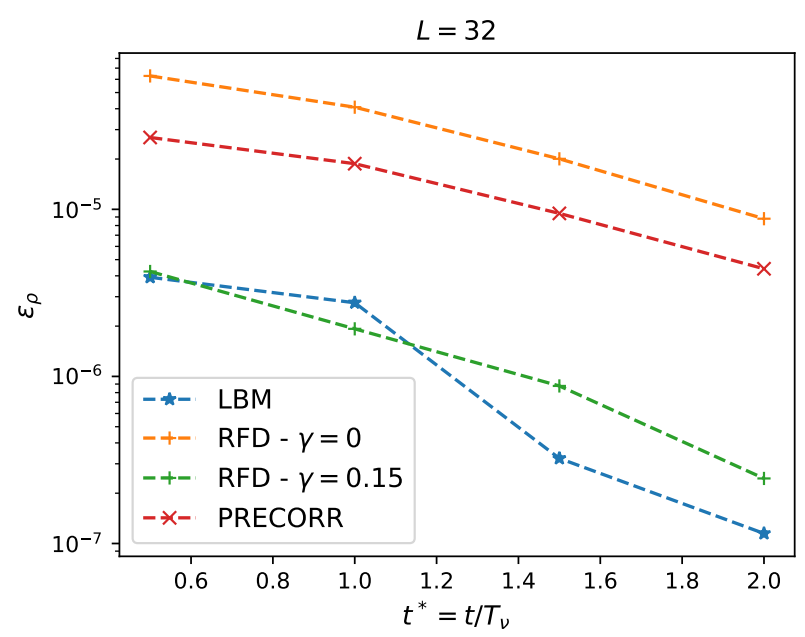

(a) $\varepsilon_{\rho}$

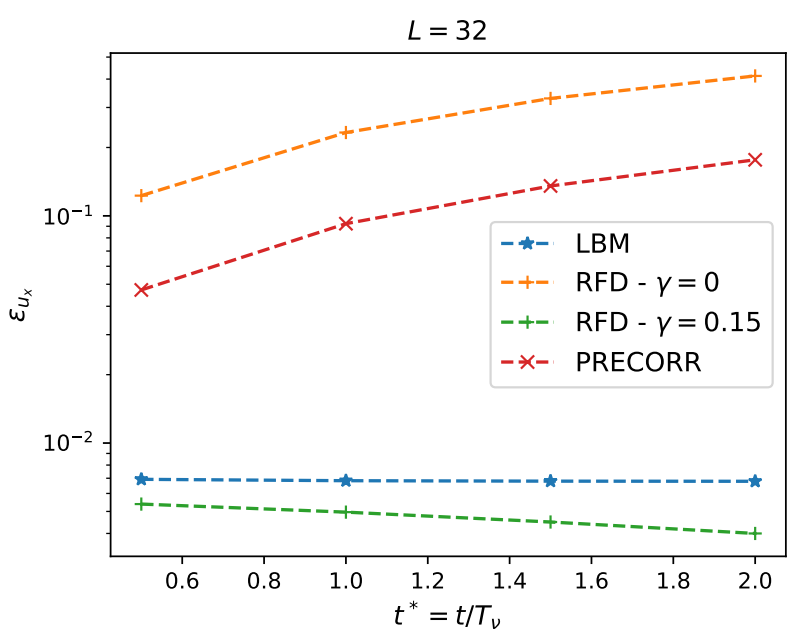

(b) $\varepsilon_{u_{x}}$

Figure 8: Taylor-Green vortex flow simulations. $L^{2}$-norm (relative) error according to the normalized time $t^{*} \equiv t / T_{\nu}$ at $\operatorname{Re}=100, L=32$ and $\nu=0.01$.

In the next configuration, both the viscosity (or relaxation time) and the resolution are kept constant 
with $\nu=0.01$ and $L=32$. The impact of the characteristic velocity (or Mach number) alone is shown in Fig. 7. The relative error in density typically increases as $\mathrm{Ma}^{2}$ whereas the velocity error remains mostly identical. For $U_{0}=0.3125$, the Mach number reaches $\mathrm{Ma}=0.5$ which is detrimental to the accuracy of the LBM algorithm. The value of the optimal $\gamma$ remains almost constant. Finally, the evolution in time of the error is plotted for $L=32, \operatorname{Re}=100$ and $\nu=0.01$ in Fig. 8. The relative error in density improves in time as the level of density fluctuations decreases. On the other hand, the velocity error deteriorates with time for the RFD $(\gamma=0)$ and PRECORR algorithms as spurious numerical dissipation accumulates. Corrections to limit this over-dissipation for the PRECORR scheme (as suggested in [24]) or $\gamma \neq 0$ for the RFD scheme appears to be mandatory.

In summary, we first confirm that the PRECORR algorithm gives very satisfactory results in view of its simplicity, namely, its macroscopic character, its locality (restricted to first neighbours) and the absence of any adjustable parameter; it performs better than the RFD algorithm with $\gamma=0$. However, adjusting $\gamma$ in the RFD scheme provides a way to significantly reduce the numerical dissipation, and obtain results comparable with those given by LBM scheme, except for very low relaxation times (in lattice units) where the LBM remains superior in terms of accuracy. The selection of $\gamma$ in the RFD scheme is important, however, a prediction of the optimal $\gamma$ would be desirable to avoid any prior trial-and-error procedure. In the next section, we will see by using a von Neumann analysis that it is possible to infer (to a certain extent) the optimal value of the RFD scheme as a function of the relaxation coefficient $\bar{\tau}$.

\section{Von Neumann analysis of the recursive finite-difference lattice Boltzmann scheme}

\subsection{Comparisons}

The so-called von Neumann (stability) analysis is a procedure that is commonly used to investigate the stability and Fourier spectral properties of finite-difference schemes [31. It is here used to gain insight about the calibration of the parameter $\gamma$ in the RFD scheme. At first, the distributions $f_{\alpha}(\boldsymbol{x}, t)$ are considered as small perturbations around a stationary and uniform reference state $(\rho, \boldsymbol{U}): f_{\alpha}=\bar{f}_{\alpha}+f_{\alpha}^{\prime}$ with $\bar{f}_{\alpha}=$ $f_{\alpha}^{e q}(\rho, \boldsymbol{U})$. The fluctuating components are expressed as complex monochromatic plane waves

$$
f_{\alpha}^{\prime}=A_{\alpha} \exp (i(\boldsymbol{k} \cdot \boldsymbol{x}-\omega t))
$$

where $\omega$ and $\boldsymbol{k}$ denote respectively the (complex) pulsation and the wavevector, $A_{\alpha}$ is the amplitude of the wave. This decomposition is introduced in our scheme

$$
f_{\alpha}^{n e q[0]}=\bar{\tau}\left(\left(-\gamma+\bar{\tau}-\frac{3}{2}\right) f_{\alpha}^{e q[0]}+(3 \gamma+2(1-\bar{\tau})) f_{\alpha}^{e q[-1]}+\left(-3 \gamma+\bar{\tau}-\frac{1}{2}\right) f_{\alpha}^{e q[-2]}+\gamma f_{\alpha}^{e q[-3]}\right)
$$

by assuming that $f_{\alpha}^{e q} \simeq \bar{f}_{\alpha}+J_{\alpha \beta} f_{\beta}^{\prime}$ with the Jacobian matrix $J_{\alpha \beta}=\left(\partial f_{\alpha}^{e q} / \partial f_{\beta}\right)$ being evaluated at the reference state. This eventually yields for each $\alpha$

$$
\begin{aligned}
\left(\delta_{\alpha \beta}-J_{\alpha \beta}\right) f_{\beta}^{\prime} & =\bar{\tau}\left(\left(-\gamma+\bar{\tau}-\frac{3}{2}\right)+(3 \gamma+2(1-\bar{\tau})) \exp \left(i\left(\omega-\boldsymbol{k} \cdot \boldsymbol{c}_{\alpha}\right) \delta_{t}\right)\right. \\
& \left.+\left(-3 \gamma+\bar{\tau}-\frac{1}{2}\right) \exp \left(2 i\left(\omega-\boldsymbol{k} \cdot \boldsymbol{c}_{\alpha}\right) \delta_{t}\right)+\gamma \exp \left(3 i\left(\omega-\boldsymbol{k} \cdot \boldsymbol{c}_{\alpha}\right) \delta_{t}\right)\right) J_{\alpha \beta} f_{\beta}^{\prime}
\end{aligned}
$$

with an implicit summation over the index $\beta$. This dispersion equation may be recast into a generalized eigenvalue problem by first rewriting it in a matrix form as $\left(\boldsymbol{A} \lambda^{3}+\boldsymbol{B} \lambda^{2}+\boldsymbol{C} \lambda+\boldsymbol{D}\right) \boldsymbol{f}^{\prime}=0$ with $\lambda=\exp \left(i \omega \delta_{t}\right)$ and by introducing the new vectors $\boldsymbol{g}^{\prime}=\lambda \boldsymbol{f}^{\prime}$ and $\boldsymbol{h}^{\prime}=\lambda \boldsymbol{g}^{\prime}$. Precisely, one obtains

$$
\left(\begin{array}{ccc}
-\boldsymbol{D} & \mathbf{0} & \mathbf{0} \\
\mathbf{0} & \boldsymbol{I} & \mathbf{0} \\
\mathbf{0} & \mathbf{0} & \boldsymbol{I}
\end{array}\right)\left(\begin{array}{l}
\boldsymbol{f}^{\prime} \\
\boldsymbol{g}^{\prime} \\
\boldsymbol{h}^{\prime}
\end{array}\right)=\lambda\left(\begin{array}{ccc}
\boldsymbol{C} & \boldsymbol{B} & \boldsymbol{A} \\
\boldsymbol{I} & \mathbf{0} & \mathbf{0} \\
\mathbf{0} & \boldsymbol{I} & \mathbf{0}
\end{array}\right)\left(\begin{array}{l}
\boldsymbol{f}^{\prime} \\
\boldsymbol{g}^{\prime} \\
\boldsymbol{h}^{\prime}
\end{array}\right)
$$

where $\boldsymbol{I}$ stands for a $9 \times 9$ identity matrix (in the case of a $D 2 Q 9$ lattice) and $A_{\alpha \beta}=\bar{\tau} \gamma \exp \left(-3 i \phi_{\alpha}\right) J_{\alpha \beta}$, $B_{\alpha \beta}=\bar{\tau}\left(-3 \gamma+\bar{\tau}-\frac{1}{2}\right) \exp \left(-2 i \phi_{\alpha}\right) J_{\alpha \beta}, C_{\alpha \beta}=\bar{\tau}(3 \gamma+2(1-\bar{\tau})) \exp \left(-i \phi_{\alpha}\right) J_{\alpha \beta}$ and $D_{\alpha \beta}=-\delta_{\alpha \beta}+(1+$ $\left.\bar{\tau}\left(-\gamma+\bar{\tau}-\frac{3}{2}\right)\right) J_{\alpha \beta}$ with $\phi_{\alpha}=\boldsymbol{k} \cdot \boldsymbol{c}_{\alpha} \delta_{t}$. 
The non-locality of the finite-difference stencil increases the size of the eigenvalue problem. The Jacobian of the equilibrium distributions has been derived analytically with the SymPy Python module for symbolic computation. The eigenvalue problem has been solved by using the SciPy Python module. The pulsations are eventually given by

$$
\omega \delta_{t}=-i \log (\lambda)
$$

for a given reference state $(\rho, \boldsymbol{U})$ at wavevector $\boldsymbol{k}$. By noting that $\exp (-i \omega t)=\exp (\Im(\omega) t) \cdot \exp (-i \Re(\omega) t)$, it follows that the dispersion of the wave is associated with $\Re(\omega)$ whereas the dissipation is linked to $\Im(\omega)$. To improve the readability of the plots, over-damped modes $\left(\Im\left(\omega_{\alpha}\right) / \nu<-10^{4}\right)$ have been disregarded. The dispersion and dissipation properties have been compared with those of the standard LB scheme, for which the von Neumann analysis leads to the eigenvalue problem

$$
\boldsymbol{f}^{\prime}=\lambda \boldsymbol{A}\left(\boldsymbol{I}-\frac{1}{\bar{\tau}_{g}}(\boldsymbol{I}-\boldsymbol{J})\right) \boldsymbol{f}^{\prime} \quad \text { with } \quad A_{\alpha \beta}=\exp \left(-i \boldsymbol{k} \cdot \boldsymbol{c}_{\alpha} \delta_{t}\right) \delta_{\alpha \beta}
$$

instead of Eq. (58). The pulsations obtained analytically from the isothermal Navier-Stokes equations 32 . will also be indicated in the figures.

In Fig. (9) and Fig. 10), the dispersion and dissipation rates are displayed as a function of the wavenumber for the standard LBM scheme, the Navier-Stokes equations and the proposed RFD scheme with different values of $\gamma$. The lattice units are used, i.e. $\delta_{x}=1$ and $\delta_{t}=1$. The Mach number and the relaxation time are the two independent parameters of the analysis. The Mach number is fixed at Ma $=0.1$ considered as a representative value. On the other hand, two relaxation times $\bar{\tau}=3 \cdot 10^{-3}$ (Fig. 9 ) and $\bar{\tau}=3 \cdot 10^{-1}$ (Fig. 10) are examined. In both cases, the reference velocity is horizontal and the analysis is restricted to waves propagating along the $x$-axis for simplicity. The wavenumber varies between 0 and $\pi$ in lattice units.

First, we can notice that the LB approach, which treats the fluid at an underlying level, involves more degrees of freedom than the macroscopic Navier-Stokes approach and, consequently, more modes in the von Neumann analysis. It is interesting to understand to what extent the "macroscopic information" is carried by the mesoscopic modes [33. In our case, the attention will focus on the dissipation properties, namely, how $\Im(\omega)$ compares with the theoretical prediction $-\nu k^{2}$ at wavenumber $k$. For $\bar{\tau}=310^{-3}$ in Fig. 9 . increasing the accuracy of the second-order derivative from first $(\gamma=0)$ to second-order accuracy $(\gamma=-\bar{\tau})$ has a negligible effect on the spectral properties. For $\gamma=-\bar{\tau}+1 / 3$, the dispersion behaviour of the shear mode is greatly improved for $k_{x}>\pi / 2$, however, $\Im(\omega)$ takes positive values involving an exponential growth detrimental to the stability. Therefore, improving the accuracy does not a priori help. For all $\gamma$, we observe that the level of dissipation of the RFD scheme is much higher than that of the LBM for $k_{x}>\pi / 8$, which agrees with the observations made in the test simulations of the previous section. In Fig. 10, the relaxation time is increased at $\bar{\tau}=0.3$. According to $\bar{\tau}=\sqrt{3} \nu / c_{s} \delta x$, this can be achieved by decreasing $\delta_{x}$ by a factor of 100 with all physical parameters kept constant. In that case, it is observed that the spectral behaviour of the RFD scheme is greatly improved and very comparable to the spectral behaviour of the LBM scheme for the dissipation, which is remarkable for a finite-difference scheme. Nevertheless, this comes with the price of increasing significantly the computational cost by reducing the grid spacing $\delta_{x}$. Interestingly, a non-trivial degraded behaviour is observed for $\gamma=-\bar{\tau}$ even if this value corresponds to a higher order in accuracy than $\gamma=0$. The optimization of $\gamma$ is therefore not straightforward.

The strategy of optimizing the spectral properties of finite-difference schemes has already been widely used in computational acoustics with the so-called dispersion-relation-preserving schemes [34]. In the lattice Boltzmann domain, the stability of the multiple-relaxation-time collision operator is optimized by carefully choosing the relaxation rates of moments through a von Neumann analysis [35, 36, 37]. The impact of various collision models on the linear stability has been recently reviewed in 27. In 38, parametric finitedifference schemes for the Boltzmann equation are proposed, and by selecting a well-suited parameter, the spurious numerical dissipation can be reduced. In the same vein, analytical developments are carried out in the next section to infer an optimal value of $\gamma$ with respect to the dissipation dynamics.

\subsection{Predicting the parameter $\gamma$}

The trial-and-error method used previously to find the optimal parameter $\gamma$ is efficient but can not be considered as an end in itself. A more straightforward way is desirable. In this subsection, we try to take 

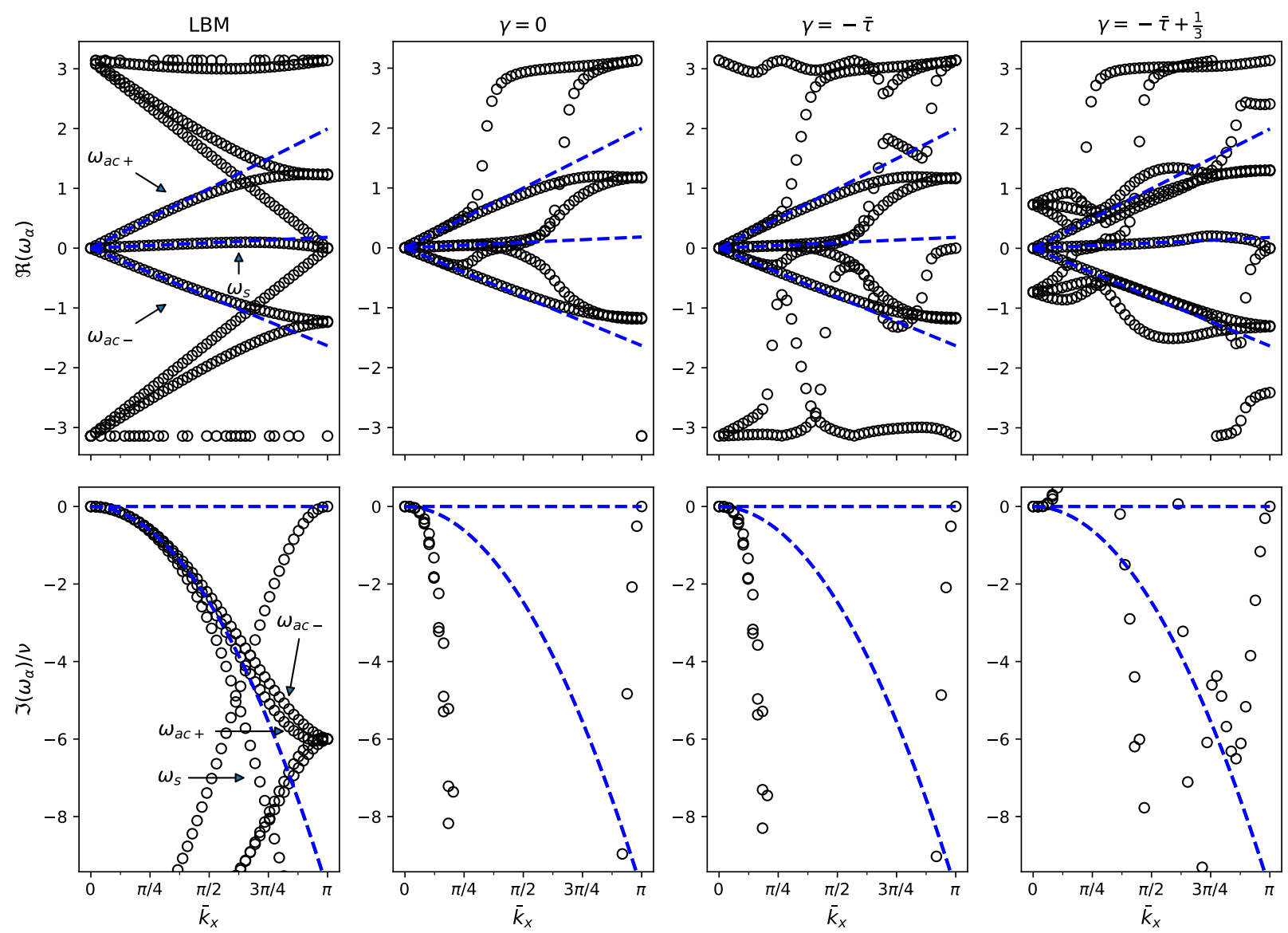

Figure 9: Von Neumann analysis: Dispersion and dissipation rates given by the real and imaginary parts of the pulsation of plane-wave perturbations around a uniform and stationary reference state at $\mathrm{Ma}=0.1$ and $\bar{\tau}=3 \cdot 10^{-3}$. Only the dependence along the $x$-axis is here considered. For the analytical Navier-Stokes solution, the different waves correspond to two acoustic modes, $\omega_{a c \pm}$ propagating at speed $U \pm c_{s}$ and a shear mode, $\omega_{s}$,propagating at speed $U$; the acoustic and shear modes are dissipated at rate $-\nu k^{2}$. In the RFD, the value $\gamma=0$ corresponds to an approximation of the first and second derivatives with second-order and first-order accuracy respectively. For $\gamma=-\bar{\tau}$ the derivatives are approximated with second-order accuracy, whereas they are approximated with third-order and second-order accuracy for $\gamma=-\bar{\tau}+\frac{1}{3}$.

advantage of the von Neumann analysis to infer the optimal $\gamma$. To allow analytical developments, we limit ourselves to the case $\mathrm{Ma}=0$. This is not restrictive since the dissipation behaviour only weakly depends on the Mach number provided that this latter remains small; the optimal $\gamma$ depends essentially on $\bar{\tau}$. The eigenvalue problem Eq. (57) can then be solved analytically by means of a computer algebra system. The shear mode is of particular interest in our case, and is related to the root of the equation

$$
\begin{array}{r}
2\left(4 \cos ^{3}\left(\bar{k}_{x}\right)-3 \cos \left(\bar{k}_{x}\right)+2\right) \gamma \bar{\tau} \lambda^{3}+\left(4 \bar{\tau} \cos ^{2}\left(\bar{k}_{x}\right)-12 \gamma \cos ^{2}\left(\bar{k}_{x}\right)-2 \cos ^{2}\left(\bar{k}_{x}\right)-6 \gamma+2 \bar{\tau}-1\right) \bar{\tau} \lambda^{2} \\
+2\left(3 \gamma \cos \left(\bar{k}_{x}\right)-2 \bar{\tau} \cos \left(\bar{k}_{x}\right)+6 \gamma-4 \bar{\tau}+2 \cos \left(\bar{k}_{x}\right)+4\right) \bar{\tau} \lambda+(-6 \gamma+6 \bar{\tau}-9) \bar{\tau}=0
\end{array}
$$

with $\lambda=\exp \left(i \omega_{s} \delta_{t}\right)$ and $\bar{k}_{x} \equiv k_{x} \delta_{x}$. From the Navier-Stokes equation, the pulsation associated with shear mode is $\omega_{s}=-i k_{x}^{2} \nu$. This pulsation is substituted in Eq. 61 by considering

$$
\lambda_{\omega_{s}}=\exp \left(\frac{\bar{k}_{x}^{2} \bar{\tau}}{3}\right)
$$



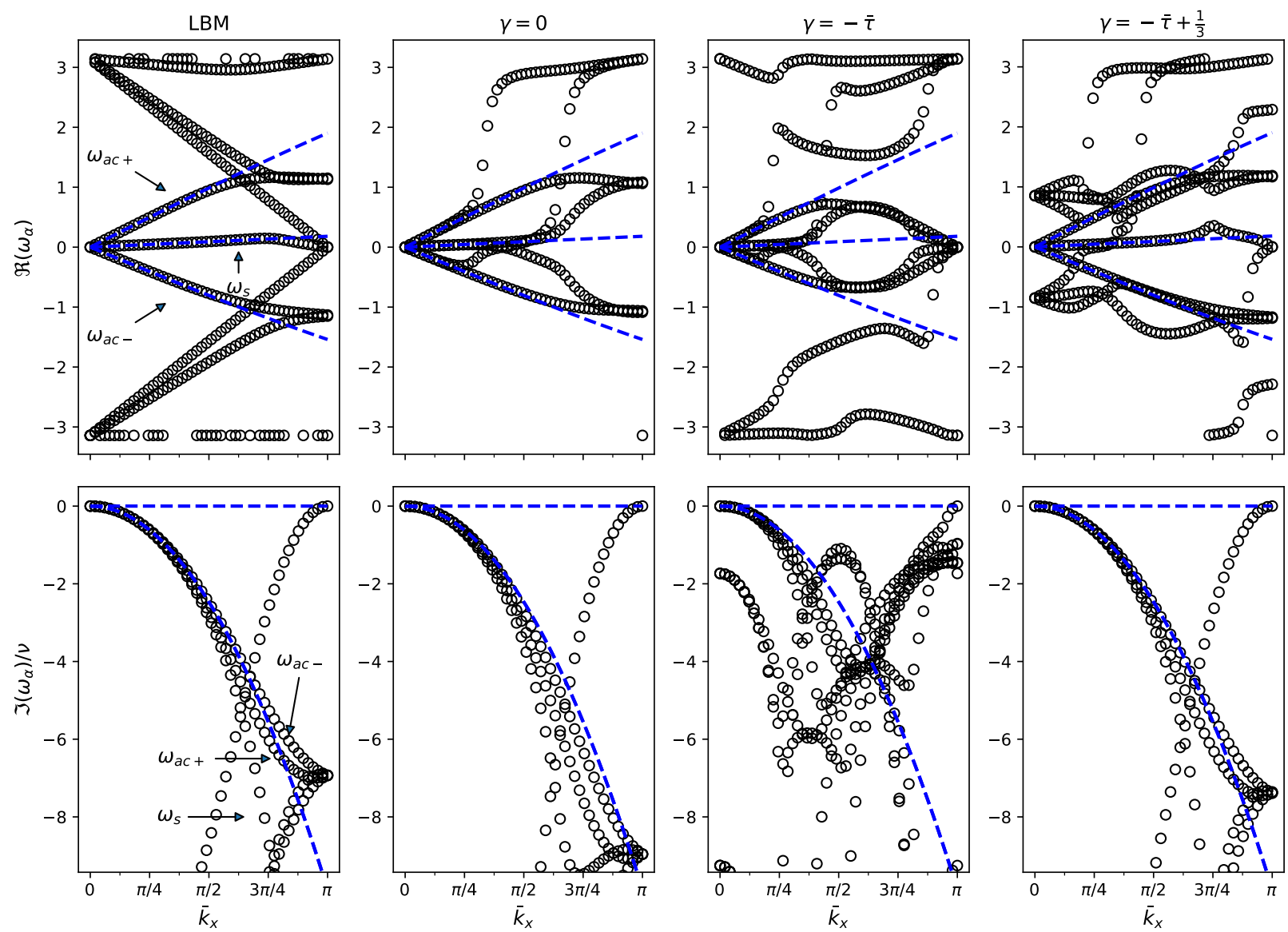

Figure 10: Von Neumann analysis: Dispersion and dissipation rates given by the real and imaginary parts of the pulsation of plane-wave perturbations around a uniform and stationary reference state at $\mathrm{Ma}=0.1$ and $\bar{\tau}=3 \cdot 10^{-1}$.

to yield

$$
\gamma\left(\bar{k}_{x}, \bar{\tau}\right)=\frac{4 A\left(\bar{k}_{x}\right)(\bar{\tau}-1) \exp \left(\frac{\bar{k}_{x}^{2} \bar{\tau}}{3}\right)+B\left(\bar{k}_{x}\right)(-2 \bar{\tau}+1) \exp \left(\frac{2 \bar{k}_{x}^{2} \bar{\tau}}{3}\right)-6 \bar{\tau}+9}{6 A\left(\bar{k}_{x}\right) \exp \left(\frac{\bar{k}_{x}^{2} \bar{\tau}}{3}\right)-6 B\left(\bar{k}_{x}\right) \exp \left(\frac{2 \bar{k}_{x}^{2} \bar{\tau}}{3}\right)+2 C\left(\bar{k}_{x}\right) \exp \left(\bar{k}_{x}^{2} \bar{\tau}\right)-6}
$$

with $A\left(\bar{k}_{x}\right)=\cos \left(\bar{k}_{x}\right)+2, B\left(\bar{k}_{x}\right)=2 \cos ^{2}\left(\bar{k}_{x}\right)+1$ and $C\left(\bar{k}_{x}\right)=4 \cos ^{3}\left(\bar{k}_{x}\right)-3 \cos \left(\bar{k}_{x}\right)+2$. The function $\gamma\left(\bar{k}_{x}, \bar{\tau}\right)$ may be interpreted as the optimal parameter $\gamma$ associated with each wavenumber $\bar{k}_{x}$ of the planewave decomposition of the solution, at a given $\bar{\tau}$. It a priori ensures that the shear mode of the RFD scheme matches exactly the shear mode of the (linearized) Navier-Stokes equations at all wavenumbers.

The colormap of $\gamma\left(\bar{k}_{x}, \bar{\tau}\right)$ and several isocontours are displayed in Fig. 11a. In practice, only an effective parameter $\gamma(\bar{\tau})$ is selected in a simulation. Therefore, the idea would be to identify a colour covering the widest range of wavenumbers at fixed $\bar{\tau}$. We see in Fig. 11a that the light-blue colour covers the range of wavenumbers $0 \leq \bar{k}_{x} \leq \pi / 4$ for $\bar{\tau} \leq 0.05$. Nonetheless, a steep variation of $\gamma$ with $\bar{k}_{x}$ is also observed at larger wavenumbers in the same range of relaxation coefficients, which indicates that approximating $\gamma\left(\bar{k}_{x}, \bar{\tau}\right)$ by a constant effective value can not be relevant at all wavenumbers. As a rule of thumb, one may claim from Fig. 11a that the scheme can only be optimized to resolve wavenumbers in the range $0 \leq \bar{k}_{x} \leq \pi / 4$ (at least eight points per wavelength) for $\bar{\tau} \leq 0.05$. The situation improves for larger relaxation coefficients, in particular when $\bar{\tau} \simeq 1 / 2$ for which $\gamma=0$ appears to be optimal at all wavenumbers. In that particular case, 


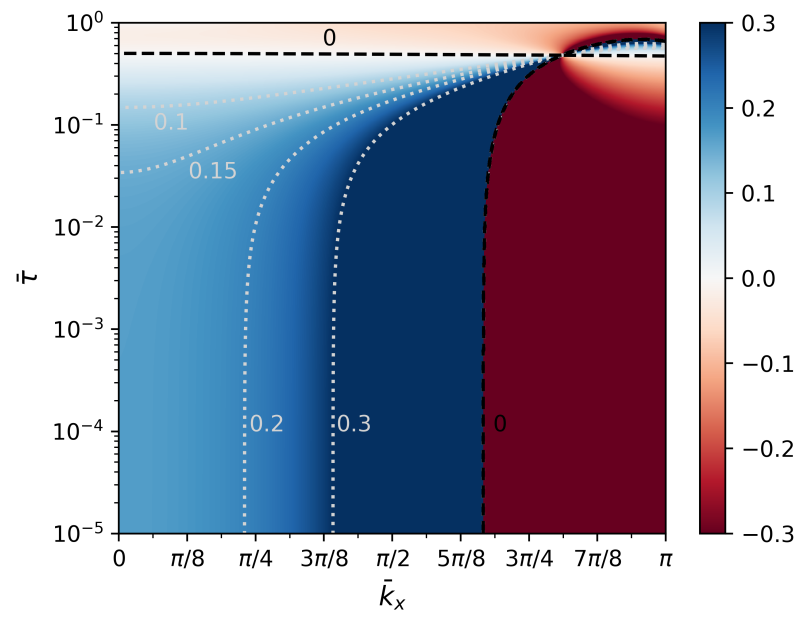

(a) Colormap of $\gamma\left(\bar{k}_{x}, \bar{\tau}\right)$ given by Eq. 63. Some isocontours are represented.
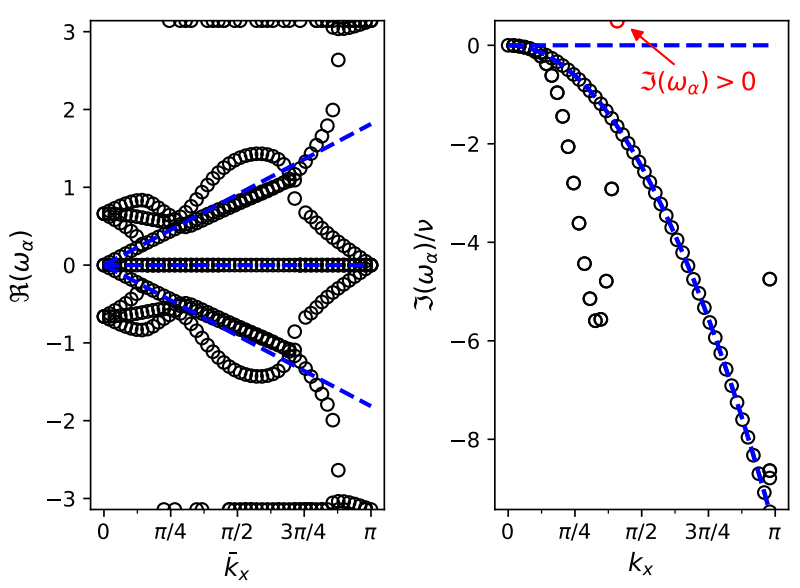

(b) Von Neumann analysis at $\mathrm{Ma}=0, \bar{\tau}=3 \cdot 10^{-3}$

Figure 11: Optimal $\gamma$ parameter at various wavenumbers and relaxation times, and its influence on the spectral properties of the scheme.

the RFD scheme consistently reduces to the LBM scheme and simply writes

$$
f_{\alpha}^{n e q[0]}=-\frac{1}{2}\left(f_{\alpha}^{e q[0]}-f_{\alpha}^{e q[-1]}\right)
$$

with $\rho(\boldsymbol{x}, t)=\sum_{\alpha} f_{\alpha}^{e q[-1]}$ and $(\rho \boldsymbol{u})(\boldsymbol{x}, t)=\sum_{\alpha} f_{\alpha}^{e q[-1]} \boldsymbol{c}_{\alpha}$. This coincidence is not surprising since the contribution of the non-equilibrium component of the distributions in the LBM scheme cancels out for the specific value $\bar{\tau}=1 / 2\left(\bar{\tau}_{g}=1\right)$. However, such favourable regime is only attainable for flows at very low Reynolds numbers or by greatly increasing the resolution.

As expected, the dissipation rate of the shear mode displays the correct behaviour in Fig. 11b. Nevertheless, we also observe that an acoustic mode triggers an instability at $\bar{k}_{x} \geq \pi / 3$ thus showing that optimizing the dissipation at all wavenumbers while preserving the stability turns out to be unrealistic. As already mentioned, a more accessible goal seems to find an effective $\gamma$ to optimize the dissipation at low wavenumbers only. In this regard, we can mention that

$$
\lim _{\bar{k}_{x} \rightarrow 0} \gamma\left(\bar{k}_{x}, \bar{\tau}\right)=\frac{1}{3} \bar{\tau}^{2}-\frac{1}{2} \bar{\tau}+\frac{1}{6}
$$

provides a lower bound in the limit of vanishing $\bar{k}_{x}=k_{x} \delta_{x}$.

In general, the value of the optimal $\gamma$ should depend on the spectral decomposition of the simulated flow. If $\bar{k}^{*}$ represents the characteristic energy-carrying wavenumber of the solution, $\gamma\left(\bar{k}^{*}, \tau\right)$ is expected to be relevant for the optimal $\gamma$ provided that $0 \leq \bar{k}^{*} \leq \pi / 4$. The values obtained by the trial-and-error method are compared with the present analytical modeling in Fig. 12. In the case of the Taylor-Green vortex simulation, one can consider that the kinetic energy is concentrated at wavenumber $\bar{k}^{*} \approx 2 \pi / L$ from the analytical form of the initial condition. The resolutions $L=32,16$ and 8 thus give respectively $\bar{k}^{*} \approx \pi / 16, \pi / 8$ and $\pi / 4$ (in lattice units). We observe in Fig. 12 that the experimental values obtained by trial-and-errors method are in excellent agreement with the theoretical prediction $\gamma\left(\bar{k}^{*}, \bar{\tau}\right)$ by varying $\bar{\tau}$ for each level of resolution. Nonetheless, we observe an increasing discrepancy with $\bar{\tau}$ that we attribute to errors in Mach number; the prediction is made at $\mathrm{Ma}=0$. The optimal $\gamma$ decreases slightly with the velocity. For the double-shear-layer configuration, the solution involves fluid structures at different scales (see Fig. 3) which implies a much broader spectral decomposition. Different resolutions are considered with $L=128,256$ and 512. The Mach and Reynolds numbers are kept constant so that the viscosity (or $\bar{\tau}$ ) 
increases proportionally to $L$. We observe that the optimal $\gamma$ obtained by the trial-and-error method lies in the interval $0.18-0.20$ corresponding to a spectral decomposition typically in the range of wavenumbers $\pi / 8-\pi / 4$. This is consistent with the number of lattice nodes used to resolve the fluid structures. The expected decrease of the optimal $\gamma$ with the resolution $L$ is also captured. However, we are not able to predict finely the optimal $\gamma$ but only to provide an order of magnitude related to the spectral domain (in wavenumbers) of the solution.

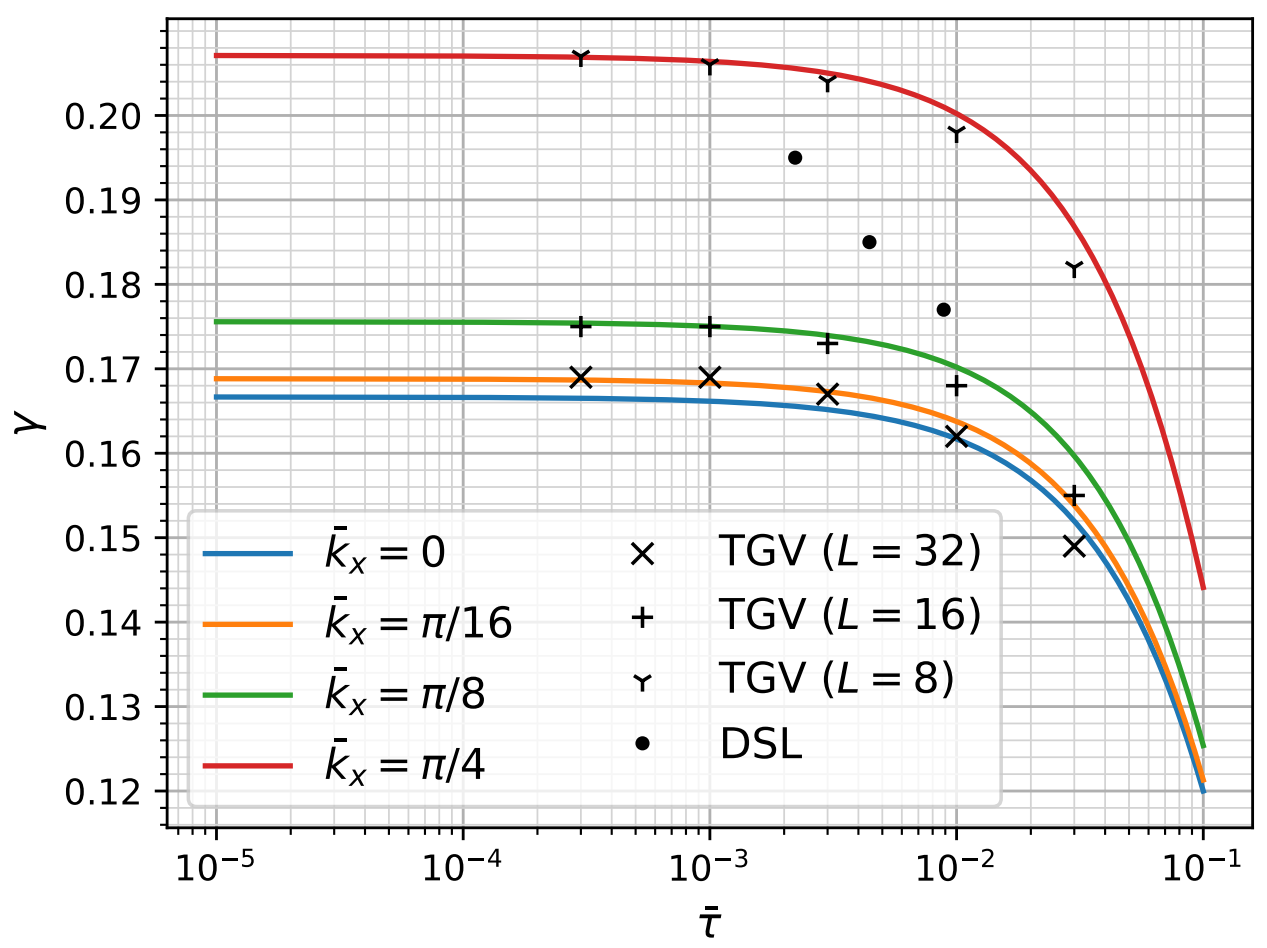

Figure 12: The lines correspond to the theoretical (von Neumann analysis) optimal $\gamma\left(\bar{k}_{x}, \bar{\tau}\right)$ as a function of $\bar{\tau}$ for various wavenumbers $\bar{k}_{x}$ at $\mathrm{Ma}=0$. The symbols correspond to the optimal $\gamma$ obtained by the trial-and-error method (with an increment of 0.001) for the Taylor-Green vortex (TGV) and double-shear-layer (DSL) simulations.

\section{Conclusion and perspectives}

This work introduces on a rigorous basis an alternative equation to the discrete-velocity Boltzmann equation to address fluid dynamics at a kinetic level, while conforming to the Navier-Stokes dynamics at the macroscopic level (in the low-Mach-number limit). This kinetic equation expresses the full distribution functions, i.e. including the equilibrium and non-equilibrium components, in terms of the flow variables $(\rho, \boldsymbol{u})$ only. However, this comes at the cost of considering a second-order differential equation instead of the first-order discrete-velocity Boltzmann equation:

$$
f_{\alpha}=f_{\alpha}^{e q}(\rho, \boldsymbol{u})-\tau\left(\frac{\partial}{\partial t}+\mathbf{c}_{\alpha} \cdot \nabla\right) f_{\alpha}^{e q}(\rho, \boldsymbol{u})+\tau^{2}\left(\frac{\partial}{\partial t}+\mathbf{c}_{\alpha} \cdot \nabla\right)^{2} f_{\alpha}^{e q}(\rho, \boldsymbol{u})
$$

From a numerical viewpoint, a first practical application that emerges from this modeling is the possibility of designing kinetic schemes that depend only on $\rho$ and $\mathbf{u}$, and which, hopefully, would inherit the advantageous spectral properties of the original LB method. 
The discretization in space and time opens a Pandora's box of possible strategies. However, since the differential equation involves particle derivatives, it was naturally chosen to integrate it along particle trajectories and therefore resort to an underlying uniform lattice structure. In addition, backward finite differences were considered to ensure an explicit character, allowing the use of solvability conditions to obtain a scheme that depends only on flow variables. A parametric family of mass-conserving finite-difference schemes was derived. Numerical simulations in two dimensions and a von-Neumann analysis indicate that the developed schemes are consistent and stable but suffer from a level of numerical dissipation which is higher than the standard Lattice Boltzmann scheme. The von-Neumann analysis shows that this deficiency is essentially linked to the approximation by backward finite differences. An optimization of the stencil is possible to approximate the physical dissipation of the Navier-Stokes equations in the range $0<\bar{k}_{x}<\pi / 4$, i.e. with a resolution greater or equal to eight points per wavelength. However, that requires prior information about the spectral decomposition of the solution itself, which is not entirely satisfactory from a numerical point of view. The use of central differences might certainly improves the situation, however in that case, we would lose the possibility of using the solvability conditions to evolve the solution in terms of the flow variables only. The tracking of the entire distributions (including equilibrium and non-equilibrium components) would therefore be a necessary compromise.

Finally, while admitting that backward finite differences are too dissipative to propagate accurately the solution over long time intervals, the developed finite-difference scheme may nevertheless be useful to locally reconstruct some missing distributions due to a defect in the lattice connectivity, e.g. at the transition between two domains with different resolutions or near a solid boundary. It may also be of interest in hybrid simulations to match Navier-Stokes and Lattice Boltzmann schemes, by providing a map between the macroscopic variables $(\rho, \boldsymbol{u})$ and the $f_{\alpha}$ 's. This point is currently under investigation.

\section{Funding}

The authors acknowledge the funding of the french DGAC under the project OMEGA3 (DGAC/DTA/SDC $\mathrm{n}^{\mathrm{o}}$ 2018-16). This work is part of a scientific collaboration including CS-group, Renault, Airbus, Ecole Centrale de Lyon, CNRS and Aix-Marseille University.

\section{Appendix A. Navier-Stokes equations from Chapman-Enskog expansion}

At $O\left(\epsilon^{0}\right), f_{\alpha}^{(0)}=f_{\alpha}^{e q}$ which imposes

$$
\begin{gathered}
\sum_{\alpha} f_{\alpha}=\rho=\sum_{\alpha} f_{\alpha}^{(0)} \quad \longrightarrow \sum_{\alpha} f_{\alpha}^{(n)}=0 \quad \forall n \geq 1, \\
\sum_{\alpha} c_{\alpha i} f_{\alpha}=\rho u_{i}=\sum_{\alpha} c_{\alpha i} f_{\alpha}^{(0)} \quad \longrightarrow \sum_{\alpha} c_{\alpha i} f_{\alpha}^{(n)}=0 \quad \forall n \geq 1 .
\end{gathered}
$$

Taking the zeroth, first, and second moments of Eq. 10 yields

$$
\begin{aligned}
\partial_{t}^{(1)} \rho+\partial_{i}^{(1)}\left(\rho u_{i}\right) & =0, \\
\partial_{t}^{(1)}\left(\rho u_{i}\right)+\partial_{j}^{(1)} \Pi_{i j}^{e q} & =0, \\
\partial_{t}^{(1)} \Pi_{i j}^{e q}+\partial_{k}^{(1)} \Pi_{i j k}^{e q} & =-\frac{1}{\bar{\tau}} \Pi_{i j}^{(1)}
\end{aligned}
$$

with $\Pi_{i j}^{e q}=\sum_{\alpha} c_{\alpha, i} c_{\alpha, j} f_{\alpha}^{e q}=\rho u_{i} u_{j}+\rho c_{s}{ }^{2} \delta_{i j}, \Pi_{i j k}^{e q}=\sum_{\alpha} c_{\alpha, i} c_{\alpha, j} c_{\alpha, k} f_{\alpha}^{e q}$ and $\Pi_{i j}^{(1)}=\sum_{\alpha} c_{\alpha, i} c_{\alpha, j} f_{\alpha}^{(1)}$. The first two equations correspond to the Euler equations with the pressure being defined by $p=\rho c_{s}^{2}$. Similarly, computing the zeroth, first, and second moments of Eq. (11) gives

$$
\begin{aligned}
\partial_{t}^{(2)} \rho & =0 \\
\partial_{t}^{(2)}\left(\rho u_{i}\right)+\partial_{j}^{(1)} \Pi_{i j}^{(1)} & =0 .
\end{aligned}
$$


By gathering the equations at $O\left(\epsilon^{1}\right)$ and $O\left(\epsilon^{2}\right)$ and multiplying each order by $\epsilon$ and $\epsilon^{2}$ respectively, one obtains

$$
\begin{aligned}
\partial_{t} \rho+\partial_{i}\left(\rho u_{i}\right) & =0 \\
\partial_{t}\left(\rho u_{i}\right)+\partial_{j}\left(\rho u_{i} u_{j}+p \delta_{i j}\right)+\partial_{j}\left(\epsilon \Pi_{i j}^{(1)}\right) & =0
\end{aligned}
$$

where

$$
\epsilon \Pi_{i j}^{(1)}=-\tau \rho c_{s}^{2}\left(\partial_{j} u_{i}+\partial_{i} u_{j}\right)+\tau \partial_{k}\left(\rho u_{i} u_{j} u_{k}\right)
$$

arises from Eqs. (A.3), (A.4) and (A.5) with the equilibrium function $f_{\alpha}^{e q}$ given by Eq. (5). The first term in Eq. A.10) suitably represents a Newtonian viscous stress with dynamic viscosity $\tau \rho c_{s}^{2}$. However, the second term indicates that the Navier-Stokes equations are not exactly recovered. This discrepancy stems from a lack of isotropy of the discrete set of microscopic velocities, here associated with the D2Q9 lattice. This lattice is not capable to represent the third-order moment $\Pi_{i j k}^{e q}$ correctly [39. Developing the equilibrium functions to an higher order in Hermite expansion does not solve the problem; only off-diagonal terms vanish. The only remedy would be to resort to a lattice with a much higher level of connectivity, e.g. the D2Q21 lattice with 21 velocities. In practice, the error term is assumed as negligible (compared to the viscous stress) provided that the Mach number remains sufficiently small.

\section{References}

[1] X. Shan, X. He, Discretization of the velocity space in the solution of the Boltzmann equation. Phys. Rev. Lett. 80 (1998) 65-68. doi:10.1103/PhysRevLett.80.65 URL http://link.aps.org/doi/10.1103/PhysRevLett.80.65

[2] T. Krüger, H. Kusumaatmaja, A. Kuzmin, O. Shardt, G. Silva, E. M. Viggen, The Lattice Boltzmann Method: Principles and Practice, Springer, 2016.

URL https : //www . springer.com/la/book/9783319446479

[3] Succi, Sauro, Lattice boltzmann 2038 EPL 109 (5) (2015) 50001. doi:10.1209/0295-5075/109/50001 URL https : //doi .org/10.1209/0295-5075/109/50001

[4] C. Körner, T. Pohl, U. Rüde, N. Thürey, T. Zeiser, Parallel Lattice Boltzmann Methods for CFD Applications, Vol. 51, Springer, 2006, Ch. 13, pp. 439-466. doi:10.1007/3-540-31619-1_13

[5] T. Inamuro, A lattice kinetic scheme for incompressible viscous flows with heat transfer Philosophical Transactions of the Royal Society of London. Series A: Mathematical, Physical and Engineering Sciences 360 (1792) (2002) $477-484$. doi:10.1098/rsta.2001.0942. URL https://doi.org/10.1098/rsta.2001.0942

[6] P. Asinari, T. Ohwada, E. Chiavazzo, A. F. D. Rienzo, Link-wise artificial compressibility method Journal of Computational Physics 231 (15) (2012) 5109-5143. doi:10.1016/j.jcp.2012.04.027 URL https://doi.org/10.1016/j.jcp.2012.04.027

[7] Z. Chen, C. Shu, D. Tan, Three-dimensional simplified and unconditionally stable lattice boltzmann method for incompressible isothermal and thermal flows, Physics of Fluids 29 (5) (2017) 053601. doi:10.1063/1.4983339 URL https://doi.org/10.1063/1.4983339

[8] D. J. Holdych, D. R. Noble, J. G. Georgiadis, R. O. Buckius, Truncation error analysis of lattice boltzmann methods, Journal of Computational Physics 193 (2) (2004) 595-619. doi:10.1016/j.jcp.2003.08.012 URL https://doi.org/10.1016/j.jcp.2003.08.012

[9] W. Zhao, W.-A. Yong, Maxwell iteration for the lattice boltzmann method with diffusive scaling Physical Review E 95 (3) (Mar. 2017). doi:10.1103/physreve.95.033311

URL https : //doi.org/10.1103/physreve.95.033311

[10] H. Otomo, B. M. Boghosian, F. Dubois, Two complementary lattice-boltzmann-based analyses for nonlinear systems Physica A: Statistical Mechanics and its Applications 486 (2017) 1000-1011. doi:10.1016/j.physa.2017.06.010 URL https://doi.org/10.1016/j.physa.2017.06.010

[11] X. Shan, X. Yuan, H. Chen, Kinetic theory representation of hydrodynamics: a way beyond the navier-stokes equation. Journal of Fluid Mechanics 550 (-1) (2006) 413. doi:10.1017/s0022112005008153 URL https://doi.org/10.1017/s0022112005008153

[12] P. L. Bhatnagar, E. P. Gross, M. Krook, A model for collision processes in gases. i. small amplitude processes in charged and neutral one-component systems, Phys. Rev. 94 (1954) 511-525. doi:10.1103/PhysRev.94.511

URL https://link.aps.org/doi/10.1103/PhysRev.94.511

[13] F. Nannelli, S. Succi, The lattice boltzmann equation on irregular lattices, Journal of Statistical Physics 68 (1992) $401-407$. doi:10.1007/BF01341755

[14] A. Bardow, I. V. Karlin, A. A. Gusev, General characteristic-based algorithm for off-lattice boltzmann simulations, Europhysics Letters (EPL) 75 (3) (2006) 434-440. doi:10.1209/epl/i2006-10138-1 URL https://doi .org/10.1209/epl/i2006-10138-1 
[15] D. Patil, Chapman-enskog analysis for finite-volume formulation of lattice boltzmann equation Physica A: Statistical Mechanics and its Applications 392 (12) (2013) 2701-2712. doi:https://doi.org/10.1016/j.physa.2013.02.016. URL https://www.sciencedirect.com/science/article/pii/S037843711300174X

[16] L. Zhu, P. Wang, Z. Guo, Performance evaluation of the general characteristics based off-lattice boltzmann scheme and dugks for low speed continuum flows, Journal of Computational Physics 333 (12 2016). doi:10.1016/j.jcp.2016.11.051

[17] G. Di Ilio, B. Dorschner, G. Bella, S. Succi, I. V. Karlin, Simulation of turbulent flows with the entropic multirelaxation time lattice boltzmann method on body-fitted meshes, Journal of Fluid Mechanics 849 (2018) 35-56. doi:10.1017/jfm. 2018.413

[18] C. Shu, Y. Wang, C. Teo, J. Wu, Development of lattice boltzmann flux solver for simulation of incompressible flows, Advances in Applied Mathematics and Mechanics 6 (2014) 436-460. doi:10.4208/aamm.2014.4.s2

[19] G. Di Ilio, D. Chiappini, S. Ubertini, G. Bella, S. Succi, Hybrid lattice boltzmann method on overlapping grids, Physical Review E 95 (01 2017). doi:10.1103/PhysRevE.95.013309

[20] J. T. Horstmann, T. Le Garrec, D.-C. Mincu, E. Lévêque, Hybrid simulation combining two space-time discretization of the discrete-velocity boltzmann equation Journal of Computational Physics 349 (2017) 399-414. doi:https://doi.org/ $10.1016 / j \cdot j c p .2017 .08 .029$

URL https://www.sciencedirect.com/science/article/pii/S0021999117306009

[21] D. Wilde, A. Krämer, K. Küllmer, H. Foysi, D. Reith, Multistep lattice boltzmann methods: Theory and applications, International Journal for Numerical Methods in Fluids 90 (3) (2019) 156-169. doi:10.1002/fld.4716 URL https://doi.org/10.1002/fld.4716

[22] J. Latt, B. Chopard, Lattice boltzmann method with regularized pre-collision distribution functions, Mathematics and Computers in Simulation 72 (2) (2006) 165-168, discrete Simulation of Fluid Dynamics in Complex Systems. doi:https: //doi.org/10.1016/j.matcom.2006.05.017

URL https://www.sciencedirect.com/science/article/pii/S0378475406001583

[23] Z. Chen, C. Shu, Simplified lattice boltzmann method for non-newtonian power-law fluid flows International Journal for Numerical Methods in Fluids 92 (1) (2019) 38-54. doi:10.1002/fld.4771. URL https://doi.org/10.1002/fld.4771

[24] Z. Chen, C. Shu, On numerical diffusion of simplified lattice boltzmann method, International Journal for Numerical Methods in Fluids (Feb. 2020). doi:10.1002/fld.4823 URL https://doi.org/10.1002/fld.4823

[25] J. Lu, H. Lei, C. Shu, C. Dai, The more actual macroscopic equations recovered from lattice boltzmann equation and their applications, Journal of Computational Physics (2020) 109546doi:10.1016/j.jcp.2020.109546 URL https://doi.org/10.1016/j.jcp.2020.109546

[26] H. Chen, X. Shan, Fundamental conditions for n-th-order accurate lattice boltzmann models Physica D: Nonlinear Phenomena 237 (14) (2008) 2003 - 2008, euler Equations: 250 Years On. doi:https://doi.org/10.1016/j.physd.2007. 11.010

URL http://www.sciencedirect.com/science/article/pii/S0167278907004137

[27] C. Coreixas, G. Wissocq, B. Chopard, J. Latt, Impact of collision models on the physical properties and the stability of lattice boltzmann methods Philosophical Transactions of the Royal Society A: Mathematical, Physical and Engineering Sciences 378 (2175) (2020) 20190397. doi:10.1098/rsta.2019.0397 URL https://doi.org/10.1098/rsta.2019.0397/

[28] P. J. Dellar, Bulk and shear viscosities in lattice boltzmann equations, Physical Review E 64 (3) (Aug. 2001). doi: 10.1103/physreve.64.031203. URL https://doi.org/10.1103/physreve.64.031203

[29] C. Coreixas, G. Wissocq, G. Puigt, J.-F. Boussuge, P. Sagaut, Recursive regularization step for high-order lattice boltzmann methods Physical Review E 96 (3) (Sep. 2017). doi:10.1103/physreve.96.033306

URL https://doi.org/10.1103/physreve.96.033306

[30] R. Mei, L.-S. Luo, P. Lallemand, D. d'Humières, Consistent initial conditions for lattice boltzmann simulations, Computers \& Fluids 35 (8-9) (2006) 855-862. doi:10.1016/j.compfluid.2005.08.008 URL https://doi.org/10.1016/j.compfluid.2005.08.008

[31] C. Hirsch, Chapter 7 - consistency, stability and error analysis of numerical schemes in: C. Hirsch (Ed.), Numerical Computation of Internal and External Flows (Second Edition), second edition Edition, Butterworth-Heinemann, Oxford, 2007, pp. 283 - 335. doi:https://doi.org/10.1016/B978-075066594-0/50049-7

URL http://www.sciencedirect.com/science/article/pii/B9780750665940500497

[32] S. Marié, D. Ricot, P. Sagaut, Comparison between lattice boltzmann method and navier-stokes high order schemes for computational aeroacoustics, Journal of Computational Physics 228 (4) (2009) 1056-1070. doi:10.1016/j.jcp.2008.10. 021 .

URL https://doi.org/10.1016/j.jcp.2008.10.021

[33] G. Wissocq, P. Sagaut, J.-F. Boussuge, An extended spectral analysis of the lattice boltzmann method: modal interactions and stability issues, Journal of Computational Physics 380 (2019) 311-333. doi:10.1016/j.jcp.2018.12.015 URL https://doi.org/10.1016/j.jcp.2018.12.015

[34] C. K. Tam, J. C. Webb, Dispersion-relation-preserving finite difference schemes for computational acoustics Journal of Computational Physics 107 (2) (1993) 262-281. doi:10.1006/jcph.1993.1142 URL https://doi.org/10.1006/jcph.1993.1142

[35] P. Lallemand, L.-S. Luo, Theory of the lattice boltzmann method: Dispersion, dissipation, isotropy, galilean invariance, and stability, Physical Review E 61 (6) (2000) 6546-6562. doi:10.1103/physreve.61.6546 
URL https://doi.org/10.1103/physreve.61.6546

[36] H. Xu, P. Sagaut, Optimal low-dispersion low-dissipation LBM schemes for computational aeroacoustics, Journal of Computational Physics 230 (13) (2011) 5353-5382. doi:10.1016/j.jcp.2011.03.040

URL https://doi.org/10.1016/j.jcp.2011.03.040

[37] M. Chávez-Modena, E. Ferrer, G. Rubio, Improving the stability of multiple-relaxation lattice boltzmann methods with central moments Computers \& Fluids 172 (2018) 397-409. doi:10.1016/j.compfluid.2018.03.084

URL https://doi.org/10.1016/j.compfluid.2018.03.084

[38] G. V. Krivovichev, Parametric schemes for the simulation of the advection process in finite-difference-based singlerelaxation-time lattice boltzmann methods, Journal of Computational Science 44 (2020) 101151. doi:10.1016/j.jocs. 2020.101151

URL https://doi.org/10.1016/j.jocs.2020.101151

[39] P. J. Dellar, Lattice boltzmann algorithms without cubic defects in galilean invariance on standard lattices Journal of Computational Physics 259 (2014) 270-283. doi:https://doi.org/10.1016/j.jcp.2013.11.021

URL https://www.sciencedirect.com/science/article/pii/S0021999113007833 